\title{
Stimulus-responsive liposomes for biomedical applications
}

\author{
Antonia I. Antoniou ${ }^{1, \S}$, Sabrina Giofrè ${ }^{1, \S}$, Pierfausto Seneci ${ }^{1}$, Daniele Passarella ${ }^{1}$, Sara Pellegrino ${ }^{2, *}$ \\ ${ }^{1}$ Dipartimento di Chimica, Università degli Studi di Milano, Via Golgi 19, Milano, 20133, Italy \\ ${ }^{2}$ Dipartimento di Scienze farmaceutiche, DISFARM, Università degli Studi di Milano, Via Venezian 21, Milano, 20133, Italy \\ ${ }^{\S}$ Authors contributed equally \\ *Correspondence: Sara.Pellegrino@unimi.it; Tel.: +3902503 14467
}

\begin{abstract}
Liposomes are amphipathic lipidic supramolecular aggregates able to encapsulate and carry molecules of both hydrophilic and hydrophobic nature. They have been widely used as in vivo drug delivery systems since long due to their favorable features, such as synthetic flexibility, biodegradability, biocompatibility, low immunogenicity, and negligible toxicity. In recent years, the possibility to introduce chemical modifications on liposomes has paved the way to smart liposome-based drug delivery systems characterized by even more tunable and disease-directed features. In this review, the different types of chemical modifications introduced so far have been highlighted, with a particular focus on internal stimuli-responsive liposomes and pro-drug activation.
\end{abstract}

Keywords: liposomes; active targeting; internal stimuli; self-assembling; targeted drug delivery; nanocarriers; enzymeresponsive liposomes; redox-responsive liposomes; pH-responsive liposomes; hypoxia-responsive liposomes, cancer therapy

\section{Introduction}

Nanomedicine and nanopharmaceuticals are growing research areas in $21^{\text {st }}$ century. Nanotechnology can indeed address several among the major issues that hamper therapeutic efficacy, limiting bench-to-clinic translation of new developed bioactive compounds $[1,2]$. In this context, the development of nanosystems for drug delivery is particularly burgeoning $[3,4]$. Appropriate nanovehicles can significantly enhance drug efficacy through an increased delivery to the molecular target and reduction of off-target effects. In addition, an optimal system should preserve drug activity and stability, protect against degradation, control drug release, and easily ensure drug excretion. A large number of drug delivery systems (DDs) has been developed so far, spanning between synthetic polymeric micelles [5], polymersomes [6], virus-like nanoparticles [7], protein [8] and peptide [9,10] nanomaterials, inorganic frameworks [11], and liposomes [12]. All of them are produced using "bottom-up" approaches, taking advantage of self-assembly (SA). SA can be defined as an "autonomous organization of components into ordered pattern or structures", and represents an elegant approach to fabricating nanostructures [13-15]. During SA, non-covalent interactions take part in defining the final features of the obtained nanomaterial. We are particularly interested in the design and synthesis of self-assembling drug-conjugates to modulate the properties of the obtained nanoassemblies against cancer and neurodegeneration $[16,17]$.

Within DDs, liposomes (LPs) possess several special advantages, being able of encapsulating both hydrophilic and lipophilic molecules, and being highly chemically versatile. Liposomes are characterized by other interesting features: synthetic flexibility, biodegradability, biocompatibility, low immunogenicity and negligible toxicity [18].

LPs are lipidic carriers that can self-assemble in water forming a membrane-like double layer that creates spherical vesicles in which an aqueous volume is enclosed [19]. Furthermore, the double layer can switch from an ordered state to a disordered state upon temperature increase [20]. This phase transition temperature $\left(T_{m}\right)$ is dependent on the molecular structures of lipids, influences LPs permeability, and thus drug release. LPs are mainly composed of phospholipids, together with other components as cholesterol and sphingolipids. The first evidence of phospholipid SA was mentioned by Bangham 
et al. in 1965 [21], reporting that phospholipids in water can form closed bilayer structures; few years later the first LP-based drug delivery system was developed. Since then several FDA-approved LPs have been introduced in therapy, and many others are in clinical trials [22]. Nowadays, LP research aims to active targeting, i.e. smart vehicles that can lead to accumulation of bioactive compounds specifically at a disease site $[23,24]$. LPs can indeed be modified on their surface by attachment of ligands or antibodies that are recognized by specific receptors [25]. On the other hand, controlled release [26] could be achieved using chemically modified lipidic components that respond to internal [27-29] or external stimuli [30]; our review here will focus on endogenous stimuli-responsive ones. Such smart LPs can thus undergo triggered drug release based on various physiology-dependent properties, allowing a better accuracy in the timing and location of payload release, and a better control over its delivery.

In this review, we report the more recent advances on chemically modified LPs, particularly focusing on cancer treatment, which are organized in four sections: 1. Redox-sensitive LPs; 2. Enzyme-sensitive LPs; 3. pH-triggered LPs; 4. Hypoxia-triggered LPs.

\section{Redox-sensitive LPs}

Among stimuli-responsive LPs, redox-sensitive ones play an important role in the field of triggered drug delivery [31]. Electron-transfer reactions may serve as controlled drug release from liposomal carriers, destabilizing their structure by changes in charge and hydrophilicity, by removal of cross-links, or by destruction of the native conformation of the amphiphile, destabilizing the LP. The development of LPs with a disulfide-bond-linked sheddable shell has recently attracted particular attention, ought to their relative stability in blood and to the fast and complete release of their payload at the targeted-site after structural disassembly of the outer core. These systems mostly rely upon the presence of tripeptide glutathione (GSH) in cellular compartments. The concentration of GSH in tumor cells is between 0.5-10 mM, i.e. 100- to 1000-fold higher than in physiological conditions [32-34]. A controlled payload release could be obtained by the reductive action of cysteine (Cys) [35].

Several chemical strategies were developed for redox-sensitive LPs, mostly based on the use of disulfide bonds as linker between phospholipids, cholesterol (Chol) and lipid-like structures, and an external hydrophilic portion (Fig. 1). Disulfide bond cleavage may disrupt LPs, releasing the payload in the intracellular environment. Other methods have been investigated, such as GSH-mediated retro-Michael-reaction of aryl thiols, reduction of quinones in cancer cells that overexpress $\mathrm{NAD}(\mathrm{P}) \mathrm{H}$ :quinone oxido-reductase isozyme 1 (NQO1), or oxidation of $\mathrm{Fe}(\mathrm{II})$ to $\mathrm{Fe}(\mathrm{III})$ in the ferrocene structure mediated by the tumor redox environment.

\section{Please insert here Figure 1}

\section{Redox-sensitive LPs based on disulfide-bonds}

A common approach in the engineering of redox-sensitive LPs foresees the insertion of a disulfide bond, which can be cleaved in a reductive environment, within the lipid or the amphiphile forming a LP. On the other hand, PEGylation was proven to be a valuable method to prevent LP clearance mediated by the reticuloendothelial system (RES), and to enhance their accumulation in tumor tissues by enhanced permeability and retention (EPR) effects thanks to the increased circulation time, resulting in passive tumor targeting. Nevertheless, the presence of a PEG shield prevents intracellular uptake, resulting in overall inefficiency of drug delivery, known as "PEG dilemma". Thus, the preparation of PEGylated LPs cleavable in response to a stimulus may be a viable solution, offering noteworthy advantages and paving the way to different approaches [36-38].

\section{Redox-triggered LP bilayer modifications}

A straightforward procedure foresees a disulfide bond linking PEG to the lipids forming the LP. As to that, extensive work has been reported by He's group, using phospholipids, compound $\mathbf{1}[35,39]$ or Chol-derived compound 2 [40-42] (Fig. 2).

Please insert here Figure 2 
In the former case, LP uptake was increased by TAT, a cell-penetrating peptide (CPP) known for its cell internalization ability. TAT was attached to the distal end of shorter PEGylated phospholipids (1,2-dioleoyl-sn-glycero-3phosphoethanolamine-DOPE-PEG 1600 -TAT or 1,2-distearoyl-sn-glycero-3-phosphorylethanolamine-DSPE-PEG $2 k$-TAT), through the sulfhydryl-maleimide reaction [39]. Thus, thiolytic PEG-deshielding of a reduction-sensitive phospholipid at the target site, markedly accelerated by the addition of exogenous Cys, exposes TAT, increasing liposomal cellular uptake (Fig. 2).

The same group further improved LP features, by replacing the phospholipid moiety with a more stable modified Chol residue 2 as a stimuli-sensitive material [40,41]. Chol-SS-PEG5k was synthetized in order to assemble a LP endowed with prolonged circulation time in vivo thanks to PEG. Herein, PEG cleavage also exposed an R8 CPP, located at the distal end of a shorter Chol-PEG2k-R8 residue, improving the therapeutic outcome with such modified LPs. Masking of positively charged CPPs is usually necessary in order to avoid their nonspecific binding in plasma, to increase their circulation time and to avoid self-aggregation. With this approach, a LP containing either doxorubicin (DOX) or the P-gp inhibitor Verapamil has been developed [41] with an encapsulation efficiency $(E E \%)$ around 94 and $70 \%(w / w)$, respectively. LPs showed good cytotoxic effects on MCF-7/ADR tumor cells.

Cluster of differentiation 44 (CD44)-targeted LPs were developed as treatment for osteosarcoma (OS). Cationic LPs were prepared and stabilized with PEG conjugated Chol derivative $\mathbf{2}$ through a reduction-sensitive disulfide linker (Chol-SS-mPEG) [42]. Hyaluronic acid (HA), a negatively charged endogenous polysaccharidic CD44 ligand, was non-covalently coated on cationic LPs $(\zeta=-28.9 \mathrm{mV}$ for HA-coated LPs). Interestingly, Chol-SS-PEG/HA-LPs were also destabilized in acidic (pH 5-6) conditions, possibly due to shedding of the HA layer, resulting in a reduced affinity for the cationic liposomal bilayers. Significant payload release ( $\approx 60 \%)$ was observed in presence of $10 \mathrm{mM} \mathrm{GSH}$.

Against OS, chitooligosaccharides (COS)-engineered LPs were developed by Wu $[43,44]$. Even if PEGylation offers many advantages, it causes low cellular uptake, slow release from endosomes and induction of anti-PEG IgM antibodies which result in accelerated blood clearance $(A B C)$. A valuable alternative is $C O S$, a derivative of chitosan (CS), that was chemically conjugated on the LP surface via a disulfide linker, yielding compound $\mathbf{3}$ (Fig. 2). Still targeting OS, the same group recently published a redox-cleavable, bone- and CD44-dual-targeting polymer through the conjugation of bone-targeting alendronate (ALN) with HA [45]. The disulfide bond between DSPE-PEG ${ }_{2 \mathrm{~K}}-\mathrm{COO}$-succinimide and ALN-HA carrying cystamine as a functional group (4, Fig. 2) was inserted into preformed LPs loaded with DOX. Co-administration of the internalizing RGD (iRGD) tumor-penetrating peptide enhanced tumor penetration of the LPs.

The use of LP engineered with HA has been exploited for other CD44-overexpressing neoplasms. Zhang [46] reported the delivery of interference RNA (RNAi) into breast tumor cells, in particular of survivin-siRNA which acts as a silencer of endogenous apoptosis inhibitors. A redox-responsive amphiphilic CS was built by layer-by-layer deposition onto the LP, and then by sequentially loading a survivin-sIRNA gene and permeation promoter hyaluronidase (HAase). Finally, the lipocomplex was decorated with $\mathrm{HA}$, by imine bond formation between $\mathrm{CS}$ and the aldehyde group arising from $\mathrm{NalO}_{4}-$ promoted diol oxidation. In this way, $\mathrm{pH}$ - and GSH-promoted deshielding, together with the increased drug penetration induced by HAase responsible of decreasing the intratumoral pressure and loosening the intercellular connective matrix, enhanced tumor accumulation and penetration of siRNAs.

In order to improve cellular siRNA delivery, stimuli-responsive cationic phospholipids were developed to ensure high transfection efficiency (TE) [47-49].

In 2010, the synthesis of redox-sensitive triazine-based gemini surfactant 5 (Fig. 3a) and their assembly in LPs for gene delivery were reported by Candiani's group [50].

Please insert here Figure 2

1,2-Dioleoyl-sn-glycero-3-phosphocholine (DOPC)/DOPE/5 lipoplex formulations yielded almost two-fold improved TE in MG63 cells compared to approved Lipofectamine 2000 formulations, and showed a GSH-dependent behavior. While a stable interaction between negatively charged DNA/RNA and cationic lipids allowed a better EE\% in the LP, such a stable system may interfere with nucleotide release at the target site. Thus, the development of stimuli-responsive systems which may induce structural rearrangement is fundamental in gene therapy. For that reason, MVL-DNA complexes were developed [51] by using cleavable multivalent lipids (compounds 6, Fig. 3a, featuring 2 to 5 positive charges at full $N$-protonation) with a disulfide bond between the cationic headgroup and hydrophobic tails. 
Lately, a polycationic amphiphile (7, Fig. 3a) with disulfide linkers for nucleic acid delivery was developed by Maslov [52]. Also, in this case an improved TE in HEK293 cells compared to Lipofectamine 2000 formulation was obtained.

To overcome survivin over-expression in breast cancer cells in response to paclitaxel (PTX), a novel (PTX/siRNA/SS-LP) nanosystem for co-delivery of PTX and anti-survivin siRNA was developed by using a redox-sensitive cationic oligopeptide lipid (8, Fig. 3a) [53]. Such nano-system showed high EE\% and rapid redox-responsive release (PTX release up to $50 \%, t=4$ $\mathrm{h}$, and $\geq 80 \%, t=36 \mathrm{~h}$ ). Endolysosomal escape of PTX/siRNA/SS-LPs led to a specific release of PTX and siRNA in 4T1 breast cancer cells.

Tocopherol-based lipids $\mathbf{9 , 1 0}$ (Fig. 3b) were widely used as LP-forming units in gene delivery [54,55]. In Patri's work [54] two tocopherol-based Cys-containing dicationic lipids (monomeric 9a and dimeric 9b, Fig. 3b) were synthetized and compared to the corresponding tocopherol-lacking lipids, resulting to be more efficacious in the transfection of a pCMVSPORT- $\beta$-gal plasmid.

In Zhao's work [55] cystamine was used as linker between tocopherol and the positively charged headgroup of either Lys 10a or His 10b (Fig. 3b). In vitro gene-TE of the lipoplexes, determined in HEK293 cells, gave for the His-based lipid (10b) a $\approx 4.3$ TE increase with respect to branched polyethylenimine bPEI-25k.

Another disulfide-based strategy entailed the formation of hydrogel cores with cross-linked 3D structures, which significantly improve mechanical stability, determine a unique rheological behavior and rapid stimuli-responsiveness to the resulting liposomal formulations [56]. LP-camouflaged, redox-responsive nanogels ("R-lipogels") were designed and prepared by LP-templated in situ polymerization process of acrylamide (AM) in presence of water-soluble cystine dimethacrylate (CDA). Lipogels were loaded with DOX, showing high drug loading capabilities and hemocompatibility ascribed to the excellent steric stabilization of PEGylated liposomal shells and to their negative surface charge, as confirmed by in vitro hemolysis.

\section{Redox-triggered prodrug activation}

In order to have an efficient administration of anticancer drugs with sub-optimal selectivity and bioavailability, the use of a prodrug in combination with its LP-encapsulation represents a valuable strategy to targeted drug delivery, preventing off-target liberation and resulting in lower side effects $[57,58]$. Recently, the conjugation of vitamin E with a docetaxel (DTX) derivative 11 (Fig. 3d) through a disulfide bond was reported [59].

To overcome the toxicity of taxotere ${ }^{\circledast}$, worsened by the use of tween-80 [60,61], LPs made by DTX-SS-VitE/DSPEPEG2k/phosphatidylcholine (PC) were assembled. The LPs showed $97.60 \% \mathrm{EE}, 7.09 \%$ DTX loading and $93.06 \mathrm{~nm}$ particle size, resulting to be a safe and efficient delivery system for controlled DTX release.

A similar strategy was developed for directed PTX release at the target site, taking advantage of a reductive disulfide cleavage, entailing the subsequent elimination of a self-immolative linker (SIL), as in compound 12 (Fig. 3d) [62]. PTX-SS-PC LPs showed a pH-sensitive release; in presence of GSH at pH 7.4, PTX release was slow, whereas in a weakly acidic environment ( $\mathrm{pH} 5.0$ ) the hydrolysis of PTX-SS-PC LPs led to $\approx 71.7 \%$ release of PTX.

A disulfide bond linker was recently inserted [63] between camptothecin (CPT) and glycerylphosphorylcholine with short $\left(C_{n}=3\right)$ and long $\left(C_{n}=11\right)$ chains (compound 13, Fig. 3d), to improve solubility, \% drug loading and tumor activity.

In another recent work [64], a redox-activated site not only ensured payload release but also showed exponential activation of fluorescence signals and photodynamic therapy (PDT) activity in response to a high level of intracellular GSH after endocytosis in tumor cells. In this case, a LP was created by self-assembly of the redox-responsive pyropheophorbide $a$-lipid conjugate 14 (Fig. 3c). These nano-carriers result from self-assembly of the conjugate $\mathbf{1 4}$ and co-encapsulation of the indoleamine 2,3-dioxygenase (IDO) inhibitor NLG-8189 into the lumen, using a remote-loading method (EE\% $>80 \%$, drug loading $\approx 5 \%$ wt\%), which promoted an increase of antigen-specific INF- $\gamma$ producing $\mathrm{T}$ cells, $\mathrm{CD} 8^{+} \mathrm{T}$ cells, and $\mathrm{CD} 4^{+} \mathrm{T}$ cells in tumor-bearing mice treated with both LPs and irradiation. Despite the limited requirements of PDT (a photosensitizer, a light source and oxygen), the high lipophilicity and poor pharmacokinetic behavior of most photosensitizers, and their strong toxicity in normal tissues (especially skin and eyes) after exposure to natural light limit their clinical applications. Therefore, the design of a novel PDT nano-carrier with targeted photo-activity to tumor tissues and minimal toxicity to healthy tissues represents a valuable tool. 


\section{Redox-triggered self-reporting strategies}

Besides drug activation promoted by an internal stimulus, other strategies have been developed in order to integrate drug targeting, imaging, stimuli-responsive drug release and therapeutic effects in a single nano system.

Liu and Li [65] reported a thiol-responsive liposomal drug carrier for intracellular self-reporting drug release based on the fluorescent probe 15 (PNO, Fig. 4). In presence of a thiol PNO undergoes reductive cleavage with subsequent intramolecular cyclization, affording compound 16 (ANO, Fig. 4). PNO-LPs show strong blue fluorescent emission at $459 \mathrm{~nm}$, while ANO is characterized by green emission at $515 \mathrm{~nm}$. The high cytotoxicity observed in HeLa cells for PNO-DOX-Ls, comparable to free DOX, can be ascribed to their rapid targeted-disassembly. A naphthalimide-sulfonamide-based nanosystem was developed by Zhang and Yu [66] to directly detect or monitor the reduction/reporting capability of LPs, especially intracellularly. Particularly interesting is the application of these nano-carriers in gene delivery, through the preparation of a cationic lipid 17 (Fig. 4). Besides the fluorescent probe, a perfluorinated $\left(\mathrm{C}_{8} \mathrm{~F}_{17}\right)$ chain and a Lys tripeptide were inserted as hydrophobic and positively charged domains, respectively. Lipoplexes formed by complexation of LPS and DNA showed weak fluorescence and good stability in the extracellular space, also due to the ability of fluorinated chains to avoid aspecific interactions with lipophilic biomolecules, thus preventing DNA degradation due to premature leaking. The lipoplexes could efficiently enter cells and then respond to endogenous thiols, to yield reduction-dependent green fluorescence emission.

\section{Please insert here Figure 3}

\section{Redox-sensitive LPs based on other chemical strategies}

Other redox release mechanisms have been exploited, including tioaryl bond cleavage and quinone reduction or ferrocene (Fe") oxidation.

LPs based on a quinone-DOPE lipid conjugate 18 were developed using DOPE, and PEG-DOPE as a lipid bilayer stabilizing agent [67]. Content release was triggered by dithionite reduction of the quinone headgroup to the corresponding hydroquinone, which underwent rapid cyclative elimination affording DOPE and a lactone (Fig. 5a). In vivo, quinones could be reduced and activated in tumor microenvironments overexpressing $\mathrm{NAD}(\mathrm{P}) \mathrm{H}$ :quinone oxidoreductase isozyme 1 (NQO1).

\section{Please insert here Figure 4}

In a related work by the same group [68], a detailed investigation of the effect of weakly hydrated chaotropic (disordermaking) anions versus highly hydrated kosmotropic (order-making) anions on payload release was carried out. Kosmotropic ions contribute to stabilize $\mathrm{H}$-bonding and intramolecular interactions in macromolecules, while chaotropic agents disrupt structures and destabilize solute aggregates [69]. Lyotropic (Hofmeister) cations and anions, as well as osmolytes, are present in time- and location-dependent concentrations within and in the vicinity of tumor cells. In particular, the time required for initial aggregation (i.a.)/payload release (p.r.) is significantly longer for Q-DOPE LPs in presence of the more polarizable/chaotropic/less hydrated $\mathrm{SCN}^{-}$(44 $\mathrm{min}$ i.a., $98 \mathrm{~min}$ p.r.) in comparison to the less polarizable/less chaotropic/more hydrated $\mathrm{Cl}^{-}$(34 min i.a., $42 \mathrm{~min}$ p.r.). Thus, increasing polarizability (chaotropicity) of the anion in solution resulted in a decreased initial release rate, which is correlated to the rate of aggregation and electrostatic interactions between the lipid layers ( $L_{\alpha}$ to $H_{\|}$transition).

As to ferrocene-modified phospholipids, Mauzeroll's group reported stable redox-triggered systems based on the insertion of an organometallic electroactive pendant to the phospholipid headgroup. This LP modification was firstly described [70] through a ferrocene-modified phospholipid 19a (Fig. 5b), achieved by a phospholipase D (PLD)-catalyzed transphosphatidylation reaction between a choline-bearing phospholipid and a primary alcohol, and synthetized as a redoxtriggerable molecule with higher stability compared to quinones. The length of the linker between the ferrocene-moiety and the phosphate group in 19a was optimized to prevent hydrolysis and/or $\mathrm{SN}_{2}$ reactions, with subsequent formation of phosphatidic acid and destabilization of the LP. LP unloading was strictly dependent on electron transfer rate and surface concentration of organometallic phospholipids. LPs-containing ferrocene phospholipids 19b in giant unilamellar vesicles (GUVs) were studied in vitro in 2016 [71]. Eventually, Mauzeroll [72] described the straightforward, scalable and costeffective synthesis of 19c (Fig. 5b) which reproducibly induced vesicle disassembly at $\approx 10 \%$ abundance within other phospholipids.

Another useful carrier foresees the synergistic effect of stimuli sensitive systems with a LP-stabilizing hydrogel. Such hybrid hydrogels, synthesized from PEG, showed improved tissue localization, minimized burst release, and controlled 
sequential delivery. The selective GSH-degradable function, thanks to the incorporation of specific thioether succinimide cross-links between PEG polymers and LPs via Michael addition, allowed rapid targeted drug release upon thiol-promoted hydrogel degradation for aryl lipogels, withcomplete hydrogel dissolution ( 3\%) and DOX release up to $70 \%$ at day 7 . (Fig. 5c) [73].

\section{Enzyme-sensitive LPs}

Enzyme-sensitive LPs also play an important role among internal stimuli-responsive LPs, since enzymatic activity might be associated to a particular tissue, or a selected enzyme may be found at higher concentrations at the diseased target site. In fact, diseases often show dysregulation of one or more enzymatic activities. In particular, proteases are overexpressed in tumor tissues, and assist the invasion and metastasis of tumor cells, representing a valuable target for selective drug delivery. By exploiting the structural requirements of a targeted enzyme, it is possible to design engineered LPs which release the drug at specific tumor microenvironments, preventing any side effects due to off-target LP disassembly. Thus, in the next paragraphs, protease- and phospholipase-centered approaches exploited in the last years will be discussed.

\section{Phospholipase-sensitive LPS}

Phospholipases hydrolyze phospholipids and are associated with cancer but also thrombosis, atherosclerosis, congestive heart failure, inflammation, neurodegeneration and infections [74,75]. Among them, secretory phospholipase A2 (sPLA2), overexpressed up to 22-fold in metastatic prostate tumors [76], emerged as the most targeted phospholipase for selective drug delivery. Thus, PLA2 from snake venom ( $\mathrm{SVPA}_{2}$ ) and human SPLA2 subtype IIA are the enzymes of choice in targeting studies. In particular, SPLA2-IIA requires a negatively charged LP membrane surface for its hydrolytic activity; for this reason, anionic phospholipids such as phosphaditylglycerols (PGs) are preferentially used to target sPLA2-IIA.

PLA2-mediated hydrolysis of fatty ester groups at the sn-2 position of glycerophospholipids makes this position a good site where to anchor enzyme-releasable bioactive molecules in a prodrug-like scenario. As a further consequence, such hydrolysis yields high concentrations of lysolipids and free fatty acids at the site of activation, which enhance permeability across biological membranes or - in large amounts - induce cellular toxicity by forming aggregate structures with detergentlike properties, due to their inverted corn-type features (Fig. 6b). sPLA2 favors organized lipid structures (such as bilayers) over monomeric lipids in solution, making it particularly suitable for liposomal drug delivery; while key structures are more easily defined for earlier described stimuli responsive LPs. The stability of the whole LPs towards SPLA2 has to be often considered. Indeed, the enzyme requires calcium as a cofactor, and its activity is determined by the composition, morphology, and physico-chemical properties of the lipid membrane; for this reason, a carefully tailored formulation of LPs is required.

\section{Phospholipase-triggered payload release}

In 2011, Cummings reported long-circulating, PEGylated and sterically-stabilized LPs (SSLPs) with Chol, and compared them with PLA2 sensitive LPs [77]. Using 6-carboxyfluorescin (6-CF) as a dye, their release profile in presence of sPLA2 was examined; 1,2-distearoyl-sn-glycero-3-phosphoglycerol (DSPG) and DSPE were suitably susceptible to sPLA2-mediated degradation compared with other phospholipids. Moreover, LPs with high content of anionic phospholipids may also activate the RES, further reducing their retention time. Conversely, their release profile in absence of sPLA2 showed a limited release $(<2 \%)$ over 108 hours, demonstrating their targeted disassembly by the enzyme. Later, the same group [76] evaluated the in vivo activity of SPLA2-responsive LPs compared to clinically used, long-circulating PEGylated SSLPs for the treatment of prostate cancer. Formulation with 10\% DSPE gave the best DOX-uptake profile, resulting in 2 to 5 -fold higher intracellular drug levels compared to SSLPs. The in vivo activity of responsive SPRL-E LPs, containing DSPE as a key phospholipid, was demonstrated using a mouse xenograft model of human prostate cancer.

A PLA2-sensitive hybrid LP composed of 1,2-dipalmitoyl-sn-glycero-3-phosphocholine (DPPC) and PEGylated blockcopolymer (Poloxamer 188) was developed for the treatment of lung cancer (Fig. 6a) [78].

\section{Please insert here Figure 6}

PLA2-sensitive LPs are used also for the treatment of infections. LPs encapsulated in a gelatin-CS hydrogel were reported for the treatment of infected wounds [79]. In the liposomal hydrogel, the model payload curcumin was embedded in the 
bilayer of the LPs. Lecithin used for LP assembly could be hydrolyzed by PLA2, which is abundant in wound exudates, resulting in curcumin release in and from the hydrogels. In the latter case, LPs stabilized by small charged nanoparticles adsorbed on the LP surfaces have been developed as a new class of environment-responsive LPs, stable against premature fusion and payload leakage [80]. Such developed PLA2-responsive LPs were conceived for the treatment of Helicobacter pylori (H. pylori) infections, as this bacterium is a strong PLA2 producer.

Phospholipase-triggered prodrug activation

A classical approach to develop LP-bound prodrugs, which might be activated by phospholipases and in particular by PLA2, is to attach a payload at the sn-2 position (red arrow, Fig. 6a), replacing the phospholipid acyl chain. Andersen and Mouritsen developed novel lipid-based retinoid prodrugs. All-trans retinoic acid (ATRA) is known for its anti-cancer activity on breast, prostate, and colon cancer, but suffers from low bioavailability, fast clearance from the bloodstream and low water solubility. In a first attempt [81], they reported the synthesis of a modified phospholipid carrying ATRA and retinoic acid receptor (RAR) on the sn-2 position. A C- 6 chain was later inserted between the glycerol backbone of the lipids and the ATRA or RAR moiety, in order to overcome the lack of enzymatic activity arising from the rigid nature of ATRA and RAR directly bound on the sn-2 position. The resulting ATRA-based 20a-b and RAR-based prodrugs 21a-b (Fig. 7) were completely hydrolyzed by SPLA2 within 24 hours, and were cytotoxic to cancer cells in presence of PLA2. In a following paper, the etherbased prodrug 1-O-stearyl-2-RAR-C6-sn-glycero-3-phosphoglycero 21b (Fig. 7) was assembled into a LP at different concentrations with DPPC, resulting in an increased PLA2 sensitivity for the RAR-prodrug [82].

Please insert here Figure 7

Besides retinoid structures also prostaglandins, and in particular the dienone 15-deoxy- $\Delta^{12,14}$-PGJ2 known for its antiproliferative activity in tumor cells, were connected as prodrugs at the sn-2 position of 1-ether and ester phospholipid (respectively 22a and 22b, Fig. 7), becoming an integral part of the lipophilic LP membrane [83]. In the PLA2-release test, while the ester conjugate $\mathbf{2 2 a}$ was completely degraded after 48 hours, the sn-1-ether 15 -deoxy- $D^{12,14}-P G J_{2}$ conjugate $\mathbf{2 2 b}$ was hydrolyzed only up to $70 \%$ at the same time.

Gemcitabine (GEM), a potent anticancer agent against non-small-cell lung carcinoma, pancreatic cancer, breast cancer and hepatocellular carcinoma, is rapidly metabolized in the blood by deoxycytidine deaminase to inactive $2^{\prime}, 2^{\prime}$ difluorodeoxyuridine. Zuo et al. [84] designed a biomimetic phospholipid-like amphiphilic prodrug, 1-0-octodecyl-2conjugated linoleoyl-sn-glycero-3-phosphatidyl GEM 23a (Fig. 8), which ensures a decrease in GEM deamination, a common metabolic step after GEM administration [85], and a rapid activation. As illustrated in Please insert here Figure , compound 23a underwent a preliminary cleavage mediated by PLA2 on the sn-2 position (step 1, Please insert here Figure ), releasing a highly surface-active lysophospholipid (23c) and unsaturated acid 23b. The induced internalization of 23c through LPmembrane disruption allowed the intracellular GEM-activation by PLD (step 2).

\section{Please insert here Figure 8}

In another work [86] an amphiphilic prodrug delivery system, also based on the elevated expression of PLD in cancer cells, was reported. DOX was inserted into DOPC via a trans-phosphatidylation reaction carried out using PLD from Streptomyces chromofuscus, yielding the prodrug-phospholipid construct 24 (Fig. 8). The resulting amphiphilic prodrug selfassembled into nanoparticles with uniform size and good stability during storage and circulation. In this way, pharmacokinetics and biodistribution of DOX were remarkably improved, with a selective triggered release by PLD into cancerous cells.

\section{Protease-sensitive LPS}

Protease overexpression is typical of many diseases, with an imbalance in the expression and activity of specific proteases at the organ target site. Among many protease families, metalloproteinases (MMPs) are a large group, that play a key role in the degradation of proteins belonging to the extracellular matrix. MMPs can promote tumor invasion, angiogenesis and metastasis by destroying the histological barrier during tumor invasion; for this reason, they are usually over-expressed in tumor microenvironments in comparison to normal tissues. Different MMP subtypes exists and in 
particular, extracellular MMP-2 and MMP-9 are over-expressed in multiple types of human cancers, and in several other diseases including rheumatoid arthritis and osteoarthritis. The exploitation of MMPs for tumor-specific liposomal drug release is not as straightforward as for the earlier introduced PLA2 strategy, since it is necessary to synthesize targeted lipopeptides that act as substrates for MMP cleavage/release activation, and to incorporate these lipopeptides in the LP membrane without altering its delicate structure.

Protease-triggered LP disassembly

Torchilin recently reported a dual stimuli-sensitive polymeric micelle with receptor-driven targetability for the codelivery of endogenous tumor suppressor miRNA-34a and DOX into cancer cells [87]. Despite its micellar nanostructure being not LP-related, the interesting dual strategies applied piqued our interest. Namely, two stimuli-sensitive constructs, a MMP2-sensitive DOX conjugate and a GSH-sensitive miRNA-34a conjugate (respectively $\mathbf{2 5}$ and 26,Fig. 9a), which were selfassembled in a single micellar nanostructure decorated with PEG for prolonged circulation and with a CPP (TATP) for enhanced intracellular delivery, have been developed.

A MMP-9-cleavable, collagen-mimetic lipopeptide $\mathbf{2 7}$ (Fig. 9b), consisting of repeated units of the Gly-Pro-Hyp tripeptide $(\mathrm{GPO})_{n}$, which forms nanosized vesicles with 1-palmitoyl-2-oleoyl-sn-glycero-3-phosphocholine (POPC), 1-palmitoyl-2oleoyl-sn-glycero-3-phosphoethanolamine (POPE)-SS-PEG, and cholesteryl-hemisuccinate (CHEMS) lipids, was developed by Mallik's group [88]. This assembled nanosystem possesses both enzyme- and redox-sensitive characteristics. PEG groups, which shield the peptide sequence recognized by MMP-9 in lipopeptides, were reductively removed only in response to elevated GSH levels. A subsequent MMP9-induced peptide-bond cleavage altered the stability of the lipid bilayer, leading to the release of encapsulated GEM.

A MMP-2 responsive peptide-hybrid LP (MRPL) was built via co-assembly of a MMP-2 responsive amphiphilic peptide $\mathbf{2 8}$ (Fig. 9b) and phospholipids, carrying pirfenidone (PFD) as a payload [89]. The latter should have down-regulated the expression of multiple extracellular matrix (ECM) components in tumors, with subsequent decrease in desmoplasia, and enhanced perfusion of PFD into the deep tumor tissue; in particular, treatment of pancreatic tumors and down-regulation of pancreatic stellate cells (PSCs) were targeted. In vivo analysis on PSCs/Mia-paca-2 pancreatic tumor bearing mice showed a decrease in penetration depth and tumor growth in mice treated with MRPL-PFD LPs and GEM, due to the increased PFDpromoted GEM-perfusion.

\section{Please insert here Figure 9}

In another strategy for targeted delivery Lee [90] reported an extremely stable "polymer-caged liposome coat", preventing any leak of contents from the LPs, and conferring additional resistance to physiological osmotic swelling. Bossmann's group, in order to ensure drug release only at the targeted site, proposed a polymer-caged LP sensitive to cancer-associated proteases (CAPs) [91]. A Chol-anchored graft copolymer composed of a short peptide sequence (Ser-GlyArg-Ser-Ala) for urokinase plasminogen activator (UPA) and poly(acrylic acid) (29, Fig. 9c) was synthesized and incorporated into LPs prepared at high osmolarities. The synthetized copolymer was incorporated into LPs and afterwards cross-linked with ethylenediamine. An alternative cross-linking by using the two amino residues of a GSGRSAGK uPA-targeted peptide (targeting sequence in red) was investigated, observing a good enzymatic release response and showing significant resistance to osmotic swelling and leaking of contents at physiological conditions.

Protease-triggered LP deshielding

Ji et al. developed a $\beta$-cyclodextrin ( $\beta$-CD) linked to a LP containing a peptide sequence cleavable by MMP-2 (30, Fig. 10a) [92], and antifibrosis and chemotherapeutic drugs for regulation of PSCs, involved in pathologically relevant stromal fibrosis. The $\beta$-CD portion was targeted to the stroma to inhibit the expression of collagen I and TGF- $\beta$ in PSCs, down-regulating fibrosis and decreasing the stromal barrier after MMP2-cleavage of the $\beta$-CD-conjugate. The post-cleaved LP, bearing the chemotherapeutic drug GEM, targeted and killed pancreatic tumor cells thanks to the increased perfusion ensured by PFD.

In order to improve tumor targetability and drug efficacy, dual-functional micellar nanoparticles were developed by combining MMP2-sensitive deshielding with folate receptor-mediated tumor targeting [93]. Folate receptors (FRs) are abundantly expressed on many cancer cells, including ovarian cancer, breast cancer and multiple myeloma, and represent a convenient strategy compared to antibody-based targeting for increasing cellular uptake. Two functional polymers, 


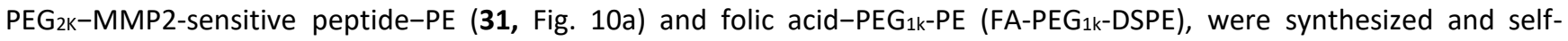
assembled with egg phopsphatidylcholine (EPC) and Chol into dual-targeted LPs. In presence of MMP-2, the long PEG ${ }_{2 K}$ chain could be removed thanks to MMP2-peptide cleavage, deshielding the FA-containing portion. In mouse models of breast cancer, FA-based LPs targeted both 4T1 breast cancer cells and M2-tumor-associated macrophages (M2-TAMs) via FAreceptor mediated endocytosis, resulting in efficient elimination of M2-TAM.

\section{Please insert here Figure10}

In order to increase target delivery through "smart" and multi-functional drug nanocarriers, decoration of LPs with various molecular entities was fully investigated [94]. Torchilin reported a nanocarrier which includes an hydrophilic and flexible long PEG, increasing the longevity of the LPs; a tumor cell-specific anti-nucleosome monoclonal antibody (mAb 2C5) anchored on a MMP-2-responsive peptidic lipid (Fig. 10b, 32a, 32b, pre- and post-modification, respectively); and a CPP (TATp)-modified phospholipid which is exposed only after long-PEG chain removal by MMP-2 enzymatic cleavage.

A novel endogenous cell-targeting nanoplatform was recently developed against idiopathic pulmonary fibrosis (IPF) through modulating M1/M2 macrophages into the suppression of fibroblast over-activation [95]. A MMP-2 responsive peptide 33 was inserted post-LP formation through Michael addition on the maleimide of a DSPE-PEG-Mal construct (Fig. 10bErrore. L'origine riferimento non è stata trovata.). The key peptide 33, grafted on the outer LP-core, consists of a linear chain of amino acid residues containing an MMP-2 specific cleavable sequence and a CXC chemokine receptor 4 (CXCR 4) targeting part, which can specifically target CXCR 4 over-expressed on the cell surface of monocyte-derived multipotent cells (MOMCs). Thus, the new MOMCs-LPs complex drove the accumulation of the same into IPF lungs, thanks to the homing ability of MOMCs. Consequently, MMP-2 sensitive release allowed the delivery of nintedanib (NIN) and colchicine (COL) at the target site, decreasing macrophage proliferation. Recently, a stimuli-responsive size-adaptable and ligand (biotin)sheddable DDS was developed making use of MMP-9 cleavable polymer-polypeptides functionalized with tumor targeting ligand biotin 34 (Fig. 10a) [96].

A fine-tuned LP for the delivery of plasmid DNA (pDNA) was constructed [97], exploiting the colloidal stability of pDNAnanoparticles in biological fluids due to PEG-coating. In order to ensure PEG-deshielding at the target site allowing a controlled payload release, $\mathrm{PEG}_{2 \mathrm{k}}$-peptidyl lipids sensitive to tumor-localized elastase or MMP-2 digestion (compounds $\mathbf{3 5}$, Fig. 10a) were co-assembled with DOPC, Chol and the cationic lipid 1,2-dioleoyl-sn-glycerol. The same group reported a similar LP-design for the development of enzymatic triggered siRNA-release [98]; using PEG 2 -peptidyl lipids 35 with peptidyl moieties sensitive to tumor-localized elastase or MMP-2 digestion. Miller et al. were able to extend the protocol for in vitro siRNA transfection into cells. Specifically, for the increased TE, the authors hypothesized either an enhanced cellular uptake mediated by peptide exposure after PEG-cleavage or an enzymes/protein-promoted cell internalization thanks to the interactions between them and the peptide moieties on LP surface.

Liu and Chen [99] reported LPs with MMPs-induced deshielding (36, Fig. 10b), with release of a compound targeting programmed death ligand 1 (PD-L1 inhibitor) after proteolytic cleavage (step 1, Fig. 10c). The authors were able to disrupt the PD-1/PD-L1 interaction, favoring an immunotherapeutic approach, based on cytotoxic T lymphocytes (CTLs) infiltrating tumor parenchyma and recognizing tumor-associated antigens. Release of DOX as a drug payload was ensured by the introduction of a pH-responsive polymer 37 (Fig. 10c), composed of dibutylpropyl amine and 1,6-hexanediol diacrylate. DOX release in acidic conditions ( $\mathrm{pH} \mathrm{5.0)}$ ) was significantly accelerated (>65\%), compared to physiological pH 7.6 (step 2, Fig. 10c).

Particularly interesting and innovative is the strategy proposed by Mizukami [100], where a two-controlled versatile nano-system was developed by using the membrane-damaging peptide temporin L (TL) and its derivatives as LP releasecontrollers. In this work protease and phosphatase activities were investigated, as a system which might be activated in response to internal stimuli. The authors synthetized a branched peptide, H-FVQWFSK(Ac-DEVD)FLGRIL-NH2, containing a substrate sequence for caspase-3 (DEVD) which could be activated upon hydrolysis. The LP membrane-damaging activity of $T L$ was controlled by caging and uncaging of Lys $\varepsilon$-amino groups; on the other hand, phosphorylation of a Tyr residue in position 8 ((H-FVQWFSKYLGRIL-NH 2 ) resulted to be optimal for achieving TL-inactivation and consequent activation upon phosphatase-mediated dephosphorylation. 


\section{Other enzyme-sensitive LPS}

Despite most published works on enzymatic-triggered LP delivery entail phospholipases-and metalloproteases as main players, different enzymes overexpressed in cancer cells and in other diseases have also been studied.

A D-alpha-tocopheryl(PEG ${ }_{1 \mathrm{k}}$ )succinate (TPGS 38, Fig. 11a) and HA dual-functionalized cationic LP containing the synthetic cationic lipid 1,5-dioctadecyl-N-histidyl-L-glutamate $\left(\mathrm{HG}_{2} \mathrm{C}_{18} 39\right)$ was developed for the co-delivery of PTX with the chemosensitizing agent Lonidamine (LND) to treat multidrug resistance cancer, by inhibition of P-gp [101]. Such sophisticated cationic LP combines different functional lipids and a HA shell, involved in the controlled release mediated by HAase (Fig. 11a). Thus, after cleavage of the shell and subsequent endolysosomal uptake, the high cationic nature of the LP combined with the proton sponge effect of the histidine imidazole groups in $\mathrm{HG}_{2} \mathrm{C}_{18}$ facilitated endolysosomal escape and cytoplasmic distribution of the LP.

\section{Please insert here Figure11}

Recently, Lou et al. [102]. developed a new liposomal platform containing a key lipid composed of a variable substrate moiety corresponding to a selected target enzyme, and acting as the trigger for LP release (40-42, Fig. 11b); a SIL located between the trigger head group and the lipid scaffold; and the lipid scaffold itself (DOPE for 40, 41 and aminodialkylglycerol for the galactosidase-responsive lipid $\mathbf{4 2}$, due to the chemical challenge associated with the introduction of the sugar head group on DOPE). While esterase- and galactosidase-based systems showed enzyme dependent release of payloads, the behavior of phosphatase-dependent LPs was completely different, with no release curve, probably due to the negative charge of the resulting LPs, given by the phosphatase- sensitive groups.

A rare example of endonuclease-triggered LP hydrogel was lately developed by Guo [103]. Acrydite-modified single strand DNA (ssDNA) was copolymerized with AM via free radical polymerization, and then hybridized with a cholesteryltetraethyleneglycol (TEG)-modified complementary DNA strand (Chol-TEG-ssDNA) using the specific recognition site (5'GAATTC- $3^{\prime}$ ) for the enzymatic cleavage mediated by the restriction endonuclease EcoR I. Hydrogel formation was afterwards achieved via mixing the DNA copolymer and the LP (DOPC) solution, thanks to the hydrophobic interaction between the cholesteryl groups of Chol-TEG-ssDNA and the lipid bilayer. The authors developed a responsive hydrogel, which was able to incorporate and release efficiently both hydrophilic and hydrophobic drugs.

Finally, a summary of redox- and enzyme-sensitive LPs is presented below in table 1.

Please insert here Table 1

\section{pH-triggered LPs}

The crucial role of the complex tumor microenvironment led to the design and development of $\mathrm{pH}$-sensitive liposomes (pHSLs), which modulate the release of their contents in response to the acidic pH environment of the tumor tissue [104]. The most common pHSLs are composed of DOPE and CHEMS [105]. DOPE is a neutral cone-shaped lipid, which shows a bilayer-to-hexagonal-phase reversible transition depending on temperature [106], whereas CHEMS contains a polar head group and is able to stabilize DOPE in bilayers. More specifically, the carboxylate head group of CHEMS is negatively charged at physiological $\mathrm{pH}$, and due to electrostatic repulsions with the positively charged amine in DOPE stabilizes DOPE molecules in a phospholipid bilayer. On the other hand, at mildly acidic pH CHEMS is protonated and DOPE molecules revert to their hexagonal structure, causing LP destabilization and release of the payload [107]. However, DOPE/CHEMS hybrid LPs are easily recognized by opsonins and driven to opsonophagocytosis [108]. To prevent their recognition by phagocytes of the RES, nanocarriers may contain pegylated phospholipids (PEG-phospholipids) to increase their in vivo half-life. Unfortunately, PEG molecules cause a decreased pH-sensitivity for DOPE/CHEMS hybrid LPs, due to stabilization of the bilayer, and also prevent intracellular penetration of the payload into tumor cells [109]. To overcome these hurdles, newly engineered pHSLPs were developed to exploit the acidic environment of the tumor cells by conjugating PEG to the surface of LPs via $\mathrm{pH}$-sensitive linkages. Suitable bonds must be stable at physiological $\mathrm{pH}$, thus providing an increased circulation half-life to the LP, but their hydrolysis at lower pH must cause PEG detachment. 


\section{pH-sensitive bonds}

Zhang et al [110] developed pHSLPs with good tumor targeting and high internalization ability at the tumor site by insertion into the LP of two modified lipids, $\mathrm{PEG}_{5 \mathrm{~K}}-\mathrm{Hz}-\mathrm{PE}\left(\mathbf{4 3}\right.$, Fig. 12) and DSPE-PEG $\mathrm{F}_{2}-\mathrm{R}_{8}$. A 5K PEG oligomer was connected to the head group of PE via 3-(2-pyridyldithiol)propionyl hydrazide, a crosslinker which contains a pH-sensitive bond. After PEG release at low extracellular $\mathrm{pH}$ conditions in tumors, the second modified lipid DSPE-PEG $2 \mathrm{k}-\mathrm{R}_{8}$ was revealed. DSPE bears on its polar head group the conjugate between a shorter, 2K PEG-maleimide and an oligopeptide. Such oligopeptide contains eight arginine units and a cysteine at the amino-terminus; the latter is used for conjugation with PEG, and contributes to LP internalization into cancer cells. Thus, the long PEGylated lipid containing a $\mathrm{Hz} \mathrm{pH}$-sensitive bond protected the peptide, due to its poor in vivo selectivity, until the LP reached the tumor cells. Then, R8 was unmasked and LPs could be internalized into tumor cells.

Similarly, Kanamala et al [111] used 1,2-dipalmitoyl-sn-glycero-3-phosphoethanolamine (DPPE) rather than DSPE, and $\mathrm{N}$-( $\varepsilon$-maleimidocaproic acid) hydrazine as a crosslinker. The modified mPEGв-Hz-DPPE lipid 44 (Fig. 12) showed good stability at $\mathrm{pH}$ 7.4, whereas protonation of the hydrazide-hydrazone bond at lower $\mathrm{pH}$ resulted in the rapid release of PEG, LP degradation and efficient drug delivery to cancer cells.

Chen et al [112] prepared and evaluated modified pHSLPs containing a cleavable double smart mPEG-lipid derivative which consisted of two ester and one $\mathrm{pH}$-sensitive hydrazone bonds. Their aim was to decrease the $\mathrm{ABC}$ phenomenon, i.e. the increased accumulation of PEG-modified LPs from the bloodstream in liver and spleen after repeated injections, by efficiently removing PEGylated coating at lower $\mathrm{pH}$ by esterase action in serum. Modified LPs containing a PEG $2 \mathrm{k}-\mathrm{Hz}-\mathrm{CHEMS}$ polymer 45 (Fig. 12) were used, and proved to be stable at physiological pH but sensitive to a mildly acidic environment (pH 5.5), where an efficient detachment of the PEG coating results from a combination of the $\mathrm{pH}$-responsive hydrazone bond hydrolysis and esterase-mediated ester bond hydrolysis. Moreover, they were less toxic and released more drug in comparison with their non-pH sensitive modified LPs (mPEG-PE).

Synthetic lipids have also been used to assemble LPs. An acylhydrazone-based acid-labile PEG ${ }_{2 k}$-lipid $\mathbf{4 6}$ (Fig. 12) was synthesized by Chan et al [113] to increase cellular uptake and endosomal escape of PEGylated cationic LP-DNA complexes.

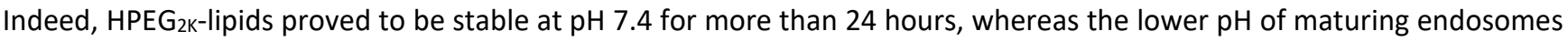
$(\mathrm{pH} 5)$ resulted in the cleavage of the PEG-coating within 1 hour.

In addition to conventional, PEGylated and CHEMS-modified LPs, Xu et al [114] showed that poly (2-ethyl-2-oxazoline) (PEtOz)-CHEMS modified LP 47 (Fig. 12) underwent acidic pH-induced increase in particle size that enhanced in vitro, $\mathrm{pH}$ dependent cellular uptake and drug release at $\mathrm{pH} 6.4$ compared to physiological $\mathrm{pH}$ 7.4. Specifically, acid-induced protonation of the amidic nitrogen resulted in the switch from a hydrophilic to a hydrophobic conformation, and destabilization of the LP. Thus, the authors believe that PEtOz and similar functionalizations could overcome the major hurdles of PEGylated polymers.

\section{Please insert here Figure12}

\section{pH-sensitive polymers and copolymers}

The straightforward preparation of nanocarriers containing $\mathrm{pH}$-sensitive polymers led to the development of novel engineered LPs for acid-responsive drug delivery. Hyperbranched poly(glycidol) (HPG) derivatives with different degrees of polymerization, such as 3-methyl-glutarylated (MGlu) HPG20-C10 (48, Fig. 12), were investigated by Yuba et al [115] as intracellular delivery vehicles. Modified MGlu-HPG-containing LPs showed high hydrophobicity and a stronger interaction with cell membranes than similar linear polymers, that was directly proportional to the increasing degree of polymerization. Furthermore, copolymers were introduced into LPs for enhanced intracellular delivery of therapeutic agents by facilitating endosomal destabilization.

Poly(styrene-co-maleic acid (SMA) (49, Fig. 12), a synthetic co-polymer introduced by Banerjee et al [116], showed a random coil conformation at physiological $\mathrm{pH}$, which collapsed and turned to a globular form at acidic $\mathrm{pH}$, resulting in the release of the LP cargo. Another copolymer was synthesized by Sugimoto et al [117] by random copolymerization of methoxy diethylene glycol methacrylate (MD-MA), a temperature-sensitive unit, with methacrylic acid (MAA), a pH sensitive unit, and lauroxy tetraethylene glycol methacrylate (LT-MA) which acted as an anchoring unit between the copolymer and egg yolk phosphatidylcholine (EYPC). MD-MAA20-LT-modified LPs $\mathbf{5 0}$ (Fig. 12) showed limited release at neutral pH, which was

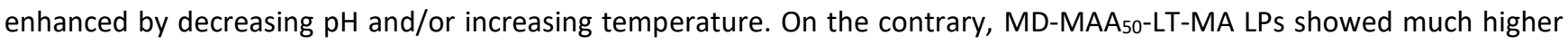


$\mathrm{pH}$ - and temperature-responsiveness at $\mathrm{pH}<6.0$ and at $\approx 45^{\circ} \mathrm{C}$, resulting in an increased cargo release due to dehydration of the diethylene glycol chains of MD-MA and to protonation of MAA carboxylates on the copolymer.

To target the delivery of a payload to a specific site, functionalized pH-sensitive LPs were designed. These LPs showed high affinity for a particular receptor due to different targeting ligands such as folate, HA, peptides, and antibodies which could also overcome the limited specificity and uptake of unmodified LPs. Lee et al [118] reported the easy fabrication of pHSLPs using hydrogenated soy phosphatidylcholine (HSPC) and HA, recognized by CD44 receptors, and conjugating them with 3-diethylaminopropyl (DEAP) units $(0.15,0.25$ and 0.40 ratios per each HA unit). HA-g-DEAP 0.40 - containing LPs showed spontaneous receptor-mediated endocytosis, fast endosomal escape, and efficient antitumor activity after protonation of $\mathrm{DEAP}$ and destabilization at $\mathrm{pH}<7.0$. Miyazaki et al [119] studied model antigenic protein ovalbumin delivery into antigenpresenting cells by $\mathrm{pH}$-sensitive HA-modified LPs. MGlu-HA and carboxycyclohexane-1-carboxylated (CHex) HA derivatives were synthesized and tested for their ability to achieve cytoplasmic delivery of antigenic proteins. CHex-HA-based LPs showed higher efficiency in the uptake and delivery of ovalbumin compared with fibroblasts, and promoted the production of Th1 cytokine from dendritic cells, resulting in therapeutic effects.

Cell membranes are negatively charged [120], so that cationic LPs are more easily internalized by cells due to their electrostatic interactions. However, they are rapidly cleared from blood circulation due to interactions with anionic biomacromolecules, such as serum components. Chen et al [121] developed cationic LPs containing Cypate, DOX and $\mathrm{NH}_{4} \mathrm{HCO}_{3}$, and shielded by a pH-responsive polymer (PSD, 51, Fig. 12), negatively charged in blood. After deshielding at lower $\mathrm{pH}$ at the tumor site, the LP surface switched to positively charged, resulting in enhanced cell internalization. After endocytosis and under NIR irradiation, LPs produced hyperthermia and rapid release of the payload. Men et al [122], using layer-by-layer methods, prepared a pH-sensitive polymer nanoparticle containing a functional LP core for drug loading, HA for increased circulation time and affinity, and poly( $\beta$-amino-ester) (PBAE) (52, Fig. 12) for controlled drug release. PBAE has been used extensively as a $\mathrm{pH}$-responsive layer, as its tertiary amine residues are protonated at acidic $\mathrm{pH}$, resulting in the release of the LP cargo at the tumor site. Specifically, the cumulative release of DOX at $\mathrm{pH} 5.0$ was more than $60 \%$ after $5 \mathrm{~h}$ and around $98.8 \%$ after $72 \mathrm{~h}$.

Furthermore, Naziris et al [123] prepared pH-responsive LPs containing structurally different chimeric LP systems made by HSPC (which exhibit phase transition temperature from gel phase $\left(L_{\beta}\right)$ to liquid crystalline phase $\left.\left(L_{\alpha}\right)[124]\right)$ and poly $(2-$ dimethylamino)ethyl methacrylate)-beta-poly(lauryl methacrylate) (PDMAEMA-beta-PLMA, 53, Fig. 12) by thin-film hydration methods. Their morphology and behaviour were dependent on biomaterial concentration, $\mathrm{pH}$ variations and polymer composition. The resulting PDMAEMA-beta-PLMA is a pH-sensitive amphiphilic diblock copolymer which responded to $\mathrm{pH}$ alterations after protonation of the amino groups of PDMAEMA at mildly acidic $\mathrm{pH}$. Moreover, PDMAEMAbeta-PLMA-modified LPs showed to be non-toxic after preliminary in vivo toxicity experiments.

\section{Zwitterionic $\mathrm{pH}$-sensitive liposomes}

Obata et al [125] investigated the influence of the head group moiety on $\mathrm{pH}$-sensitive LPs through the synthesis of the zwitterionic lipids 1,5-dihexadecyl $\mathrm{N}$-glutamyl-L-glutamate (54, Fig. 12) and 1,5-dihexadecyl $N, N$-diglutamyl-lysyl-Lglutamate (55, Fig. 12). Both modified LPs were evaluated for their $\mathrm{pH}$-responsiveness during in vitro intracellular drug delivery as well as for their in vivo anticancer efficacy compared to conventional DPPC-LPs. Such LPs showed higher intracellular drug delivery and toxicity against HeLa cancer cells. Yaroslavov et al [126] induced the assembly of conventional zwitterionic EPC with an amphoteric lithocholic acid derivative 56 (Fig. 12), containing anionic and cationic groups at each end of its steroid core. Compound $\mathbf{5 6}$ can change its orientation due to acidic/basic $\mathrm{pH}$ disordering of the lipid bilayer, thus resulting in the release of the LP cargo. Remarkably, $50-60 \%$ of an encapsulated antitumor drug (DOX and cisplatin) was released from such LPs within the first minutes after acidification of the surrounding medium.

\section{Conformationally switchable LPs and Fliposomes}

pHSLPs based on amphiphiles, known as flipids or fliposomes, can perform a pH-triggered conformation flip. Liu et al [127] incorporated trans-2-aminocyclohexanol-based lipids into LPs to provide them with a pH-sensitive conformational flip in an acidic environment (57 \& 58, Fig. 13). Enhanced leakage of the LP cargo was observed after protonation of the amine nitrogen and the formation of a strong intramolecular hydrogen bond with the hydroxy group, which led both groups to switch from axial to equatorial positions. Furthermore, the conformational switch of the polar groups forced the lipid tails to a mirror switch from equatorial to axial conformation, resulting in the destabilization of the LP membrane. Veremeeva et 
al. [128] described the synthesis of a similar molecule with two long alkyl substituents and a chair-boat conformation at $\mathrm{pH}$ $>7$, which was inserted into phosphotidylcholine-LPS. Below pH 7.0, protonation of nitrogen atoms led to a different orientation of the two alkyl chains, so that the molecule changed its conformation from chair-boat to chair-chair. As a result, reorganization of the alkyl chains caused destabilization and leakage of the LP cargo within a few minutes at pH between 57. More recently, Liu prepared a series of trans-2-aminocyclohexanol-based flipids for structure activity relationship studies [129]. After studying the basicity, polarity and size of the headgroup, and the length of the tail, they concluded that the size of the fliposome depends on its headgroup, and decreases when larger or more polar headgroups were used. On the other side, higher basicity of the headgroup may cause premature cargo release, as it could be protonated even at physiological $\mathrm{pH}$; as a result, the flipid would change its conformation before reaching more acidic targets such as endosomes (pH 5.06.5) and solid tumors ( $\mathrm{pH}$ 6.5-7.2). Moreover, higher encapsulation was achieved by longer tails, while shorter tails showed increased drug release at lower $\mathrm{pHs}$.

Please insert here Figure13

\section{LPs functionalized with $\mathrm{pH}$-sensitive peptides}

CPP-decorated, pHSLPs spanning payloads varying from nanosized particles to DNA fragments were conceived for cellular internalization [130]. Jiang et al [131] assembled a dual-decorated $\mathrm{HA}-\left(\mathrm{R}_{6} \mathrm{H}_{4}\right)$-LP for tumor-targeted drug delivery. Firstly, the HA- $\mathrm{R}_{6} \mathrm{H}_{4}-\mathrm{LP}$ was targeted to the tumor site by HA, where HAase released $\mathrm{HA}$ and exposed the $\mathrm{R}_{6} \mathrm{H}_{4}$ sequence, where arginine was responsible of cell penetration and histidine could be protonated in mildly acidic conditions, ensuring a $\mathrm{pH}$-dependent response. The synergistic effect of the two residues ensured for $\mathrm{HA}_{-} \mathrm{R}_{6} \mathrm{H}_{4}-\mathrm{LP}$ an enhanced anticancer drug delivery, therapeutic efficacy, and decreased drug toxicity. More recently, Zhao et al [132] prepared and characterized pHSLPs encapsulated with DOX for the treatment of glioma. The $\mathrm{pH}$-responsive peptide $\mathrm{H}_{7} \mathrm{~K}\left(\mathrm{R}_{2}\right)_{2}$ was introduced onto LPs as a targeting ligand which also exhibited $\mathrm{pH}$-triggered $\mathrm{DOX}$ release at the acidic $\mathrm{pH}$ environment in gliomas. Moreover, the anti-tumor activity of DOX- $\mathrm{H}_{7} \mathrm{~K}\left(\mathrm{R}_{2}\right)_{2} \mathrm{pHSLPs}$ was confirmed by in vivo experiments. Similarly, Shi et al [133] prepared a pHsensitive CPP and anchored it on the surface of LPs for delivering PTX in tumor cells with low systemic toxicity. The cyclic CRGDfK peptide, specific to overexpressed integrin $\alpha_{v} \beta_{3}$ on activated endothelial cells, was bound through an amide bond between the $\varepsilon$-amino group of lysine and the $\mathrm{C}$ terminus of the H-CLIHH(Aib)LHALHHLNIHGLLYGA-OH peptide, which contains several His residues for $\mathrm{pH}$-triggered efficient drug delivery. Cysteine at the $\mathrm{N}$-terminus acted as an anchor between the peptide and DSPE-PEG2K-maleimide. LPs bearing the c(RGDFK)-AGYLLGHINLHHLAHL(Aib)HHIL-Cys CPP exhibited strong binding and selectively targeted integrin $\alpha_{v} \beta_{3}$ overexpressing cancer cells, due to the RGD fragment of the cyclic peptide. Moreover, efficient accumulation and penetration of PTX in the tumor was observed due to the negative charge of the peptide in the blood circulation and its positive charge at the tumor side. The pH-responsive SKDEEWHKNNFPLSP peptide was investigated by Han et al [134], being an affinity ligand of the angiogenesis marker vascular endothelial growth factor receptor 2 (VEGFR2). Its pH-responsiveness is due to the KDEE-segment at its $\mathrm{N}$-terminus, which forms an $\alpha$ helix in presence of protons. Thus, efficient drug delivery and in vivo therapy could be "switched on" in a mildly acidic environment, such as where VEGFR2 is overexpressed.

\section{LPs functionalized with orthoester nucleoside lipids}

Orthoester nucleoside lipids (ONLs, 59 and 60, Fig. 14) were introduced by Oumzil et al [135] for the assembly of pH sensitive LPs. Their synthesis entailed the attachment of a dialkyl orthoester chain to the secondary hydroxy group, and of a charged or neutral hydrophilic moiety to the primary hydroxy group of thymidine; on the other side, their hydrolysis rate depends on proton concentration. Thus, such ONL-modified LPs, when exposed to the acidic tumor microenvironment around $\mathrm{pH}$ 5.0, showed an increased hydrolysis rate in comparison with $\mathrm{pH}$ 7.4. At the lower, acidic $\mathrm{pH}$ LP destabilization took place through orthoester bond hydrolysis, followed by the attachment of the hexadecanol to the DOPC. This led to cone-shaped complexes and to the formation of a hexadecanoyl/DOPC lamellar system, which promoted LP destabilization and the release of their cargo. ONL-based LPs efficiently delivered siRNAs targeted against human RecQ helicase (RECQL4) for the treatment of breast cancer. 


\section{LPs functionalized with unnatural $\mathrm{pH}$-sensitive lipids}

The preparation and characterization of a pH-sensitive pentaerythritol phosphatidylcholine (PEPC) lipid (61, Fig. 14) with marginal toxicity was described by Zhan et al [136]. This lipid was synthesized via latentiation in good yields with a trialkoxybenzylidene acetal group as a pH-sensitive moiety. Self-assembly of PEPC lipids in aqueous solution led to the formation of LPs, which were loaded with fluorescent calcein to prove their $\mathrm{pH}$-sensitivity. These smart drug carriers were stable at $\mathrm{pH} 7.4$, whereas they showed size increase and $80 \%$ release of their cargo within 5 hours at pH 4.5 due to the cleavage of the cyclic acetal. Liskayová et al [137] investigated the relationship between the structure of $N, N$ dimethylalkane-1-amine $\mathrm{N}$-oxides 62 ( $\mathrm{C}_{\mathrm{n}} \mathrm{NO}, \mathrm{n}=1-18$, Fig. 14$)$ and in vitro TE of pH-sensitive DNA- $\mathrm{C}_{\mathrm{n}} \mathrm{NO} / \mathrm{DOPE}$ lipoplexes (cationic, non-viral lipid-DNA complexes) by delivering plasmid DNA (pEGFP-N1) into human bone OS epithelial cells (U2OS). Zeta potential measurements and small angle X-ray scattering showed dependence of the $\mathrm{C}_{n} \mathrm{NO}$ structure on the complex's composition and on $\mathrm{pH}$. More specifically, a conformational transition from cubic $\left(\mathrm{Q}_{11}\right)$ and lamellar $\left(\mathrm{L}_{\mathrm{a}}\right)$ phases at neutral $\mathrm{pH}$ to condensed lamellar $\left(\mathrm{La}^{\mathrm{c}}\right)$ and condensed inverted hexagonal $\mathrm{H}_{11}^{\mathrm{c}}$ phases at acidic $\mathrm{pH}$ was observed. $\mathrm{C}_{16} \mathrm{NO} / \mathrm{DOPE}$ was identified as the most effective LP, with a 3-fold higher transfection efficiency than commercial Lipofectamine 2000.

Please insert here Figure14

\section{Hypoxia-triggered liposomes}

Hypoxia is the disrupted balance between the supply and consumption of oxygen, observed in many solid tumors [138] and leading to resistance to radiotherapy [139], chemotherapy [140], and to poor prognosis [141]. Feng et al [142], by exploiting the hypoxic tumor microenvironment in combination with non-invasive, light-triggered cancer PDT, developed PEG-shelled theranostic LPs encapsulated with the hydrophobic photosensitizer chlorin e6 and the hydrophilic bioreductive prodrug AQ4N (63a, Fig. 15), activated by hypoxia inhibiting topoisomerase II. AQ4N-hCe6-LPs proved to be versatile imaging probes for in vivo tracking by photoacoustic (PA)-, fluorescence- and positron emission tomography (PET)-imaging; moreover, ex vivo experiments confirmed a promising nanocarrier activity as anticancer therapies.

Furthermore, Liu et al [143] developed a synergistic chemo-/ radiotherapy treatment for malignant glioma by preparing a hypoxic radiosensitizer LP for the delivery of DOX to the tumor. Hypoxic nitroimidazole radiosensitizers were conjugated through a hydrolysable ester bond with hexadecanedioic acid, using 3-(dimethylamino)-1,2-propanediol as a linker between the two fatty acids (MDH, 64a, Fig. 15) to prepare hypoxia-dependent LPs. These LPs showed both in vitro and in vivo enhanced intracellular uptake, and efficient release of a DOX payload under hypoxic conditions. In details, reduction of the poorly water soluble, hydrophobic nitroimidazole derivative 64 a to the corresponding highly water-soluble hydrophilic aminoimidazole derivative $\mathbf{6 4 b}$ led to LP destabilization and, eventually, to payload release. Besides, such LPs overcame the resistance of cancer cells to radiation due to their intra-tumoral hypoxia, and provided permeability through the otherwise impermeable blood-brain barrier. Similarly, Li et al [144] introduced a nitroimidazole derivative 65a (Fig. 15) for the construction of hypoxia-triggered LPs. Such LPs also demonstrated a hypoxia-dependent payload release both in cellular assays and in xenograft models implanted with clinically relevant patient cell lines. Due to their hydrophobic character and their ability to convert to the corresponding hydrophilic aminoimidazoles via a series of selective bioreductions, nitroimidazoles have been used widely as nanocarriers in the development of hypoxia-triggered LPs.

Unnatural azo-inserted lipids containing Si rather than $\mathrm{P}$ atoms were reported by Long et al (66a, Fig. 15) [145]. The corresponding hybrid LPs selectively released in vitro and in vivo their payload close to tumor sites, after responding to overexpressed hypoxia inducible factor $1 \alpha$ (HIF-1 $\alpha$ ).

\section{Please insert here Figure15}

Finally, a summary of $\mathrm{pH}$ - and hypoxia-sensitive LPs is presented below in table 2 .

Please insert here Table2 


\section{Conclusions and future perspectives}

Different chemical modifications introduced in the last years in the development of endogenous responsive LPs were presented in this review. Specifically, these nano-DDS significantly increase the choice of treatments for common diseases, in particular in chemotherapy, where the selective release of hazardous drugs may notably reduce toxicity issues and offtarget effects. An internal stimuli-triggered release is based on tailored-sensitive molecules, which respond to different environmental conditions. Namely, the presence of specific enzymes and the mild acidic pH in tumor microenvironments cause their selectivity. However, a premature disruption of the LP and the leakage of its cargo before reaching the target sites, together with substantial individual differences among patients are drawbacks often observed in these systems. Herein, many approaches used to answer such central issues in the development of responsive-LPs have been reported, to give an outlook on this topic and at the same time, to offer a landmark to selectively choose among the tailor-made molecules for building multifunctional LPs.

In particular, recent trends point toward the construction of multifunctional DDS able to expose active targeting molecules on cue, as well as to release different drugs sequentially, thanks also to the presence of penetration enhancers. In our opinion, this synergistic combination of different approaches and of multiple release mechanisms could be the future direction for the development of new LP-based DDs. The further introduction of self-reporting systems, which allow a realtime measurement of the efficiency of a given therapy by the determination of the biodistribution and site accumulation of the drug in the body, could also represent a smart strategy for answering the individual response to therapy, allowing further progress toward personalized interventions.

\section{Acknowledgments}

This study was implemented in the frame and with the financial support of the research project "New frontiers of engineered nanovectors to improve treatment efficacy and safety in neurological - NEVERMIND Project."

Conflicts of Interest: The authors declare no conflict of interest.

Abbreviations: $A B C$, accelerated blood clearance; $A L N$, alendronate; $A M$, acrylamide; $A Q 4 N, \quad 1,4-b i s[[2-$ (dimethyloxidoamino)ethyl]amino]-5,8-dihydroxy-9,10-anthracenedione dihydrochloride; $\beta$-CD, $\beta$-cyclodextrin; CHEMS, cholesteryl-hemisuccinate; Chex-HA, carboxycyclohexane-1-carboxylated hyaluronic acid; Chol, cholesterol; CLSM, confocal laser scanning microscopy; CPPs, cell-penetrating peptides; CPT, camptothecin; CS, chitosan; DEAP, 3-diethylaminopropyl; DDS, drug delivery systems; DMPE, 1,2-dimyristoyl-sn-glycero-3-phosphoethanolamine; DOPE, 1,2-dioleoyl-sn-glycero-3phosphoethanolamine; DOX, doxorubicin; DOPC, 1,2-dioleoyl-sn-glycero-3-phosphocholine; DPPC, 1,2-dipalmitoyl-snglycero-3-phosphocholine; DPPE, 1,2-dipalmitoyl-sn-glycero-3-phosphoethanolamine; DSPE, 1,2-Distearoyl-sn-glycero-3phosphorylethanolamine; DSPG, 1,2-distearoyl-sn-glycero-3-phosphoglycerol; DTX, docetaxel; EPC, egg phosphatidylcholine; EPR, enhanced permeability and retention; EYPC, egg yolk phosphatidylcholine; GSH, glutathione; HA, hyaluronic acid; hCe6, hydrophobic chlorin e6; HSPC, hydrogenated soy phosphatidylcholine; HSPEC, hydrogenated soy phosphatidylcholine; $\mathrm{Hz}$, hydrazone bond; LPs, liposomes; LT-MA, lauroxy tetraethylene glycol methacrylate; MAA, methacrylic acid; MD-MA, methoxy diethylene glycol methacrylate; MGlu-HPG, 3-methyl-glytarylated hyperbranched poly(glycidol);MMP, matrix metalloproteinase; MPEG, methoxy polyethylene glycol; $\mathrm{MPEG}_{\mathrm{B}}$, methyl ether polyethylene benzaldehyde; NIR, near infrared; ONL, orthoester nucleoside lipid; OS, osteosarcoma; PBAE, poly( $\beta$-amino-ester); PDMAEMA-beta-PLMA, poly(2-dimethylamino)ethyl methacrylate)-beta-poly(laury methacrylate); PE, phosphatidylethanolamine; PEG, polyethylene glycol; PEPC, pentaerythritol phosphatidylcholine; PET, positron emission tomography; PEtOz, poly (2-ethyl-2-oxazoline); PFD, pirfenidone; pHSLs, pH sensitive liposomes; PLA, phospholipase A; POPC, 1-palmitoil-2-oleoyl-sn-glycero-3-phosphocholine; POPE, 1-palmitoyl-2-oleoyl-sn-glycero-3-phosphoethanolamine; PSCs, pancreatic stellate cells; PTX, paclitaxel; $R_{8}$, eight units of arginine; RES, reticuloendothelial system; SIL, self-immolative linker; SMA, poly(styrene-co-maleic acid); TAMs, tumor-associated macrophages; TE, transfection efficiency; TEG, tetraethylene glycol.

\section{References:}

[1] Bawa, R. (2010) Nanopharmaceuticals: Nanopharmaceuticals. Eur. J. Nanomedicine 3, 34-40. https://doi.org/https://doi.org/10.1515/EJNM.2010.3.1.34.

[2] Farjadian F. et al. (2018) Nanopharmaceuticals and nanomedicines currently on the market: challenges and 
opportunities. Nanomed. J. 14, 93-126. https://doi.org/10.2217/nnm-2018-0120.

[3] Eftekhari, R.B. et al. (2019) Co-delivery nanosystems for cancer treatment: A Review. Pharm. Nanotechnol. 7, 90112. https://doi.org/http://dx.doi.org/10.2174/2211738507666190321112237.

[4] Qin, X., Li, Y. (2020) Strategies to design and synthesize polymer-based stimuli-responsive drug-delivery nanosystems. Chem. Bio. Chem. 21, 1236-1253. https://doi.org/https://doi.org/10.1002/cbic.201900550.

[5] Yousefpour Marzbali, M., Yari Khosroushahi, A. (2017) Polymeric micelles as mighty nanocarriers for cancer gene therapy: a review. Cancer Chemother. Pharmacol. 79, 637-649. https://doi.org/10.1007/s00280-017-3273-1.

[6] Zhang, X., Zhang, P. (2017) Polymersomes in nanomedicine - A Review. Curr. Nanosci. 13, $124-129$. https://doi.org/http://dx.doi.org/10.2174/1573413712666161018144519.

[7] Kong, J. et al. (2018) Rationally designed peptidyl virus-like particles enable targeted delivery of genetic cargo. Angew. Chem. Int. Ed. 57, 14032-14036. https://doi.org/10.1002/anie.201805868.

[8] Peng, Q., Mu, H. (2016) The potential of protein-nanomaterial interaction for advanced drug delivery. J. Control Release 225, 121-132. https://doi.org/https://doi.org/10.1016/j.jconrel.2016.01.041.

[9] Bucci, R. et al. (2017) Self-assembly of an amphipathic $\alpha \alpha \beta$-tripeptide into cationic spherical particles for intracellular delivery. Org. Biomol. Chem. 15, 6773-6779. https://doi.org/10.1039/C70B01693J.

[10] Bonetti, A. et al. (2015) Dipeptide nanotubes containing unnatural fluorine-substituted $\beta 2,3$-diarylamino acid and Ialanine as candidates for biomedical applications. Org. Lett. 17, 4468-4471. https://doi.org/10.1021/acs.orglett.5b02132.

[11] Baeza, A., Ruiz-Molina, D., Vallet-Regí, M. (2017) Recent advances in porous nanoparticles for drug delivery in antitumoral applications: inorganic nanoparticles and nanoscale metal-organic frameworks. Expert Opin. Drug Deliv. 14, 783-796. https://doi.org/10.1080/17425247.2016.1229298.

[12] Alavi, M., Karimi, N., Safaei, M. (2017) Application of various types of liposomes in drug delivery systems. Adv. Pharm. Bull 7, 3-9. https://doi.org/10.15171/apb.2017.002.

[13] Bucci, R. et al. (2020) Nucleobase morpholino $\beta$ amino acids as molecular chimeras for the preparation of photoluminescent materials from ribonucleosides. Sci. Rep. 10, 19331. https://doi.org/10.1038/s41598-020-762977.

[14] Clerici, F., Erba, E., Gelmi, M.L., Pellegrino, S. (2016) Non-standard amino acids and peptides: From self-assembly to nanomaterials. Tetrahedron Lett. 57, 5540-5550. https://doi.org/https://doi.org/10.1016/j.tetlet.2016.11.022.

[15] Fumagalli, G. et al. (2016) Self-assembly drug conjugates for anticancer treatment. Drug Discov. Today 21, 13211329. https://doi.org/https://doi.org/10.1016/j.drudis.2016.06.018.

[16] Colombo, E. et al. (2020) New class of betulinic acid-based nanoassemblies of cabazitaxel, podophyllotoxin, and thiocolchicine. ACS Med. Chem. Lett. 11, 895-898. https://doi.org/10.1021/acsmedchemlett.9b00668.

[17] Colombo, E. et al. (2019) Nanolipid-trehalose conjugates and nano-assemblies as putative autophagy inducers. Pharmaceutics 11, 422. https://doi.org/10.3390/pharmaceutics11080422.

[18] Sercombe, L. et al. (2015) Advances and challenges of liposome assisted drug delivery. Front. Pharmacol. 6, 286.

[19] A kbarzadeh, A. et al. (2013) Liposome: classification, preparation, and applications. Nanoscale Res. Lett. 8, 102. https://doi.org/10.1186/1556-276X-8-102.

[20] Chen, W. et al. (2018) Determination of the main phase transition temperature of phospholipids by nanoplasmonic sensing. Sci. Rep. 8, 14815. https://doi.org/10.1038/s41598-018-33107-5.

[21] Bangham, A.D., Standish, M.M., Watkins, J.C. (1965) Diffusion of univalent ions across the lamellae of swollen phospholipids. J. Mol. Biol. 13, 238-252. https://doi.org/10.1016/S0022-2836(65)80093-6.

[22] Bulbake, U., Doppalapudi, S., Kommineni, N., Khan, W. (2017) Liposomal formulations in clinical use: An updated review. Pharmaceutics 9. https://doi.org/10.3390/pharmaceutics9020012.

[23] Bazak, R. et al. (2015) Cancer active targeting by nanoparticles: a comprehensive review of literature. J. Cancer Res. Clin. Oncol. 141, 769-784. https://doi.org/10.1007/s00432-014-1767-3.

[24] Yan, W., Leung, S.S.Y., To, K.K.W. (2019) Updates on the use of liposomes for active tumor targeting in cancer therapy. Nanomedicine (Lond.) 15, 303-318. https://doi.org/10.2217/nnm-2019-0308.

[25] Riaz, M.K. et al. (2018) Surface functionalization and targeting strategies of liposomes in solid tumor therapy: A review. Int. J. Mol. Sci. 19, 195. https://doi.org/10.3390/ijms19010195.

[26] Heidarli, E., Dadashzadeh, S., Haeri, A. (2017) State of the art of stimuli-responsive liposomes for cancer therapy. Iran J. Pharm. Res. 16, 1273-1304. https://doi.org/10.22037/ijpr.2017.2164.

[27] Lee, Y., Thompson, D.H. (2017) Stimuli-responsive liposomes for drug delivery. Wiley Interdiscip. Nanomed. Nanobiotechnol. 9. https://doi.org/https://doi.org/10.1002/wnan.1450.

[28] Nultsch, K., Germershaus, O. (2018) Matrix metalloprotease triggered bioresponsive drug delivery systems - Design, 
synthesis and application. Eur. J. Pharm. Biopharm. 131, 189-202. https://doi.org/10.1016/j.ejpb.2018.08.010.

[29] Mu, J., Lin, J., Huang, P., Chen, X. (2018) Development of endogenous enzyme-responsive nanomaterials for theranostics. Chem. Soc. Rev. 47, 5554-5573. https://doi.org/10.1039/c7cs00663b.

[30] Wang, Y., Kohane, D.S. (2017) External triggering and triggered targeting strategies for drug delivery. Nat. Rev. Mater. 2, 17020. https://doi.org/10.1038/natrevmats.2017.20.

[31] Sun, H. et al. (2013) Reduction-sensitive degradable micellar nanoparticles as smart and intuitive delivery systems for cancer chemotherapy. Expert Opin. Drug Deliv. 10, 1109-1022. https://doi.org/10.1517/17425247.2013.783009.

[32] Gamcsik, M.P., Kasibhatla, M.S., Teeter, S.D., Colvin, O.M. (2012) Glutathione levels in human tumors. Biomarkers 17, 671691 https://doi.org/10.3109/1354750X.2012.715672.

[33] Cook, J.A. (2004) Oxidative stress, redox, and the tumor microenvironment. Semin. Radiat. Oncol. 14, 259-266. https://doi.org/10.1016/j.semradonc.2004.04.001.

[34] Bansal, A., Simon, M.C. (2018) Glutathione metabolism in cancer progression and treatment resistance. J. Cell Biol. 217, 2291-2298. https://doi.org/10.1083/jcb.201804161.

[35] Kuai, R. et al. (2011) Targeted delivery of cargoes into a murine solid tumor by a cell-penetrating peptide and cleavable poly(ethylene glycol) comodified liposomal delivery system via systemic administration. Mol. Pharm. 8, 2151-2161. https://doi.org/10.1021/mp200100f.

[36] Romberg, B., Hennink, W.E., Storm, G. (2008) Sheddable coatings for long-circulating nanoparticles. Pharm. Res. 25, 55-71. https://doi.org/10.1007/s11095-007-9348-7.

[37] Hatakeyama, H., Akita, H., Harashima, H. (2013) The polyethyleneglycol dilemma: Advantage and disadvantage of PEGylation of liposomes for systemic genes and nucleic acids delivery to tumors. Biol. Pharm. Bull. 36, 892-899. https://doi.org/10.1248/bpb.b13-00059.

[38] Fang, Y. et al. (2017) Cleavable PEGylation: a strategy for overcoming the "PEG dilemma" in efficient drug delivery. Drug Deliv. 24, 22-32. https://doi.org/10.1080/10717544.2017.1388451.

[39] Kuai, R. et al. (2010) Efficient delivery of payload into tumor cells in a controlled manner by TAT and thiolytic cleavable PEG Co-modified liposomes. Mol. Pharm. 7, 1816-1826. https://doi.org/10.1021/mp100171c.

[40] Tang, J. et al. (2014) A detachable coating of cholesterol-anchored PEG improves tumor targeting of cell-penetrating peptide-modified liposomes. Acta Pharm. Sin. B 4, 67-73. https://doi.org/10.1016/j.apsb.2013.12.004.

[41] Tang, J. et al. (2016) Co-delivery of doxorubicin and P-gp inhibitor by a reduction-sensitive liposome to overcome multidrug resistance, enhance anti-tumor efficiency and reduce toxicity. Drug Deliv. 23, 1130-1143. https://doi.org/10.3109/10717544.2014.990651.

[42] Chi, Y. et al. (2017) Redox-sensitive and hyaluronic acid functionalized liposomes for cytoplasmic drug delivery to osteosarcoma in animal models. J. Control Release 261, 113-125. https://doi.org/10.1016/j.jconrel.2017.06.027.

[43] Yin, X., et al. (2017) Chitooligosaccharides modified reduction-sensitive liposomes: Enhanced cytoplasmic drug delivery and osteosarcomas-tumor inhibition in animal models. Pharm. Res. 34, 21722184. https://doi.org/10.1007/s11095-017-2225-0.

[44] Yin, X. et al. (2018) Estrogen-functionalized liposomes grafted with glutathione-responsive sheddable chotooligosaccharides for the therapy of osteosarcoma. Drug Deliv. 25, 900-908. https://doi.org/10.1080/10717544.2018.1458920.

[45] Feng, S. et al. (2019) Engineering of bone- and CD44-dual-Ttargeting redox-sensitive liposomes for the treatment of orthotopic osteosarcoma. ACS Appl. Mater. Interfaces. 11, 7357-7368. https://doi.org/10.1021/acsami.8b18820.

[46] Chen, H. et al. (2020) Stimuli-responsive polysaccharide enveloped liposome for targeting and penetrating delivery of survivin-shRNA into breast tumor. ACS Appl. Mater. Interfaces. 12, 22074-22087. https://doi.org/10.1021/acsami.9b22440.

[47] Li, J. et al. (2015) A review on phospholipids and their main applications in drug delivery systems. Asian J. Pharm. Sci. 10, 81-98. https://doi.org/10.1016/j.ajps.2014.09.004.

[48] Koynova, R., Tenchov, B. (2009) Cationic phospholipids: structure-transfection activity relationships. Soft Matter. 5, 3187-3200. https://doi.org/10.1039/B902027F.

[49] Stamatatos, L., Leventis, R., Zuckermann, M.J., Silvius, J.R. (1988) Interactions of cationic lipid vesicles with negatively charged phospholipid vesicles and biological membranes. Biochemistry 27, 3917-3925. https://doi.org/10.1021/bi00411a005.

[50] Candiani, G. et al. (2010) Bioreducible liposomes for gene delivery: From the formulation to the mechanism of action. PLoS One 5, 1-8. https://doi.org/10.1371/journal.pone.0013430.

[51] Shirazi, R.S.et al. (2012) Structural evolution of environmentally responsive cationic liposome-DNA complexes with a reducible lipid linker. Langmuir 28, 10495-10503. https://doi.org/10.1021/la301181b. 
[52] Puchkov, P.A. et al. (2016) Design, synthesis and transfection efficiency of a novel redox-sensitive polycationic amphiphile. Bioorganic Med. Chem. Lett. 26, 5911-5915. https://doi.org/10.1016/j.bmcl.2016.11.005.

[53] Chen, X. et al. (2017) Co-delivery of paclitaxel and anti-survivin siRNA via redox-sensitive oligopeptide liposomes for the synergistic treatment of breast cancer and metastasis. Int. J. Pharm. 529, 102-115. https://doi.org/10.1016/j.ijpharm.2017.06.071.

[54] Kedika, B., Patri, S.V. (2012) Synthesis and gene transfer activities of novel serum compatible reducible tocopherolbased cationic lipids. Mol. Pharm. 9, 1146-1162. https://doi.org/10.1021/mp200435y.

[55] Zheng, L.T. et al. (2016) Reducible amino acid based cationic lipids as highly efficient and serum-tolerant gene vectors. ChemPlusChem 81, 125-134. https://doi.org/10.1002/cplu.201500307.

[56] Ma, J. et al. (2018) Liposomes-camouflaged redox-responsive nanogels to resolve the dilemma between extracellular stability and intracellular drug release. Macromol. Biosci. 18, 1800049. https://doi.org/10.1002/mabi.201800049.

[57] Markman, M. (2003) Management of toxicities associated with the administration of taxanes. Expert Opin. Drug Safz. 2, 141-146. https://doi.org/10.1517/14740338.2.2.141.

[58] Vaishampayan, U., Parchment, R.E., Jasti, B.R., Hussain, M. (1999) Taxanes: an overview of the pharmacokinetics and pharmacodynamics. Urology 54, 22-29. https://doi.org/10.1016/S0090-4295(99)00451-3.

[59] Ren, G. et al. (2018) Construction and cellular uptake behavior of redox-sensitive docetaxel prodrug-loaded liposomes. Pharm. Dev. Technol. 23, 22-32. https://doi.org/10.1080/10837450.2017.1287728.

[60] Immordino, ML. et al. (2003) Preparation, characterization, cytotoxicity and pharmacokinetics of liposomes containing docetaxel. J. Control Release 91, 417-429. https://doi.org/10.1016/S0168-3659(03)00271-2.

[61] Park, M.H. ey al. (2014) A novel aqueous parenteral formulation of docetaxel using prodrugs. Int. J. Pharm. 462, 17. https://doi.org/10.1016/j.ijpharm.2013.12.027.

[62] Wang, Z. et al. (2019) Reduction responsive liposomes based on paclitaxel-ss-lysophospholipid with high drug loading for intracellular delivery. Int. J .Pharm. 564, 244-255. https://doi.org/10.1016/j.ijpharm.2019.04.060.

[63] He, W. et al. (2019) Redox-sensitive dimeric camptothecin phosphatidylcholines-based liposomes for improved anticancer efficacy. Nanomedicine 14, 3057-3074. https://doi.org/10.2217/nnm-2019-0261.

[64] Liu, D. et al. (2019) Redox-activated porphyrin-based liposome remote-loaded with Indoleamine 2,3-dioxygenase (IDO) inhibitor for synergistic photoimmunotherapy through induction of immunogenic cell death and blockage of IDO pathway. Nano Lett. 19, 6964-6976. https://doi.org/10.1021/acs.nanolett.9b02306.

[65] Chen, W. et al. (2014) Self-reporting liposomes for intracellular drug release. Small 10, 1261-1265. https://doi.org/10.1002/smll.201302698.

[66] Wang, B.et al. (2018) A reduction-responsive liposomal nanocarrier with self-reporting ability for efficient gene delivery. J. Mater. Chem. B 6, 2860-2868. https://doi.org/10.1039/c8tb00392k.

[67] Loew, M., Forsythe, J.C., McCarley, R.L. (2013) Lipid nature and their influence on opening of redox-active liposomes. Langmuir 29, 6615-6623. https://doi.org/10.1021/la304340e.

[68] McCarley, R.L. (2013) Release rates of liposomal contents are controlled by kosmotropes and chaotropes. Langmuir 29, 13991-13995. https://doi.org/10.1021/la402740k.

[69] Yang Z. (2009) Hofmeister effects: an explanation for the impact of ionic liquids on biocatalysis. J. Biotechnol 144, 12-22. https://doi.org/10.1016/j.jbiotec.2009.04.011.

[70] Correia-Ledo, D., Arnold, A.A., Mauzeroll, J. (2010) Synthesis of redox active ferrocene-modified phospholipids by transphosphatidylation reaction and chronoamperometry study of the corresponding redox sensitive liposome. $J$. Am. Chem. Soc. 132, 15120-15123. https://doi.org/10.1021/ja105921g.

[71] Noyhouzer, T. et al. (2016) Ferrocene-modified phospholipid: An innovative precursor for redox-triggered drug delivery vesicles selective to cancer cells. Langmuir 32, 4169-4178. https://doi.org/10.1021/acs.langmuir.6b00511.

[72] Odette, W.L., Payne, N.A., Khaliullin, R.Z., Mauzeroll, J. (2019) Redox-triggered disassembly of nanosized liposomes containing ferrocene-appended amphiphiles. Langmuir 35, 5608-5616. https://doi.org/10.1021/acs.langmuir.8b04267.

[73] Liang, Y., Kiick, K.L. (2016) Liposome-cross-linked hybrid hydrogels for glutathione-triggered delivery of multiple cargo molecules. Biomacromolecules 17, 601-614. https://doi.org/10.1021/acs.biomac.5b01541.

[74] Sun, G.Y. (2010) Phospholipases A2 and inflammatory responses in the central nervous system. NeuroMolecular Med. 12, 133-148. https://doi.org/10.1007/s12017-009-8092-z.

[75] Olson, N. et al. (2008) Lipoprotein-associated phospholipase A2 and risk of venous thrombosis in older adults. Am. J. Hematol. 83, 524-527. https://doi.org/10.1002/ajh.21182.

[76] Quach, ND. et al. (2014) Role of the phospholipase A2 receptor in liposome drug delivery in prostate cancer cells. 
Mol. Pharm. 11, 3443-3451. https://doi.org/10.1021/mp500174p.

[77] Zhu, G. et al. (2011) Secretory phospholipase A2 responsive liposomes. J. Pharm. Sci. 100, 3146-3159. https://doi.org/https://doi.org/10.1002/jps.22530.

[78] Tagami, T., Ando, Y., Ozeki, T. (2017) Fabrication of liposomal doxorubicin exhibiting ultrasensitivity against phospholipase A2 for efficient pulmonary drug delivery to lung cancers. Int. J. Pharm. 517, 35-41. https://doi.org/10.1016/j.ijpharm.2016.11.039.

[79] Li, D., An, X., Mu, Y. (2019) A liposomal hydrogel with enzyme triggered release for infected wound. Chem. Phys. Lipids 223, 104783. https://doi.org/10.1016/j.chemphyslip.2019.104783.

[80] Thamphiwatana, S. et al. (2014) Phospholipase A2-responsive antibiotic delivery via nanoparticle-stabilized liposomes for the treatment of bacterial infection. J. Mater. Chem. B 2, 8201-8207. https://doi.org/10.1039/c4tb01110d.

[81] Pedersen, P.J. et al. (2010) Liposomal formulation of retinoids designed for enzyme triggered release. J. Med. Chem. 53, 3782-3792. https://doi.org/10.1021/jm100190c.

[82] Arouri, A., Mouritsen, O.G. (2011) Anticancer double lipid prodrugs: Liposomal preparation and characterization. J. Liposome Res. 21, 296-305. https://doi.org/10.3109/08982104.2011.563365.

[83] Pedersen, P.J. et al. (2010) Prostaglandin phospholipid conjugates with unusual biophysical and cytotoxic properties. Bioorganic. Med. Chem. Lett. 20, 4456-4458. https://doi.org/10.1016/j.bmcl.2010.06.054.

[84] Zuo, J. et al. (2017) Biomimetic nanoassemblies of 1-O-octodecyl-2-conjugated linoleoyl-sn-glycero-3-phosphatidyl gemcitabine with phospholipase A2-triggered degradation for the treatment of cancer. Colloids Surf. B Biointerfaces 152, 467-474. https://doi.org/10.1016/j.colsurfb.2017.02.001.

[85] Serdjebi, C. et al. (2013) Rapid deaminator status is associated with poor clinical outcome in pancreatic cancer patients treated with a gemcitabine-based regimen. Pharmacogenomics 14, 1047-1051. https://doi.org/10.2217/pgs.13.93.

[86] Tao, X. et al. (2017) Design and evaluation of a phospholipase D based drug delivery strategy of novel phosphatidylprodrug. Biomaterials 131, 1-14. https://doi.org/10.1016/j.biomaterials.2017.03.045.

[87] Salzano, G. et al. (2016) Mixed nanosized polymeric micelles as promoter of doxorubicin and miRNA-34a co-delivery triggered by dual stimuli in tumor tissue. Small 12, 4837-4848. https://doi.org/10.1002/smll.201600925.

[88] Kulkarni, P.S. et al. (2014) MMP-9 responsive PEG cleavable nanovesicles for efficient delivery of chemotherapeutics to pancreatic cancer. Mol. Pharm. 11, 2390-2399. https://doi.org/10.1021/mp500108p.

[89] Ji, T. et al. (2017) Designing liposomes to suppress extracellular matrix expression to enhance drug penetration and pancreatic tumor therapy. ACS Nano. 11, 8668-8678. https://doi.org/10.1021/acsnano.7b01026.

[90] Lee, S.M. et al. (2007) Polymer-caged lipsomes: A pH-responsive delivery system with high stability. J. Am. Chem. Soc. 129, 15096-15097. https://doi.org/10.1021/ja070748i.

[91] Basel, M.T., Shrestha, T.B., Troyer, D.L., Bossmann, S.H. (2011) Protease-sensitive, polymer-caged liposomes: A method for making highly targeted liposomes using triggered release. ACS Nano. 5, 2162-2175. https://doi.org/10.1021/nn103362n.

[92] Ji, T. et al. (2016) An MMP-2 responsive liposome integrating antifibrosis and chemotherapeutic drugs for enhanced drug perfusion and efficacy in pancreatic cancer. ACS Appl. Mater. Interfaces 8, 3438-3445. https://doi.org/10.1021/acsami.5b11619.

[93] Deng, C. et al. (2019) Tumors and their microenvironment dual-targeting chemotherapy with local Immune adjuvant therapy for effective antitumor immunity against breast cancer. Adv. Sci. 6, 1801868. https://doi.org/10.1002/advs.201801868.

[94] Zhu, L., Kate, P., Torchilin, V.P. (2012) Matrix metalloprotease 2-responsive multifunctional liposomal nanocarrier for enhanced tumor targeting. ACS Nano. 6, 3491-3498. https://doi.org/10.1021/nn300524f.

[95] Chang, X. et al. (2020) Nanoengineered immunosuppressive therapeutics modulating M1/M2 macrophages into the balanced status for enhanced idiopathic pulmonary fibrosis therapy. Nanoscale 12, 8664-8678. https://doi.org/10.1039/dOnr00750a.

[96] Jin, Y. et al. (2020) Size-adaptable and ligand (biotin)-sheddable nanocarriers equipped with avidin scavenging technology for deep tumor penetration and reduced toxicity. J. Control Release 320, 142-158.m https://doi.org/10.1016/j.jconrel.2020.01.040.

[97] Yingyuad, P. et al. (2013) Enzyme-triggered PEGylated pDNA-nanoparticles for controlled release of pDNA in tumors. Bioconjugz. Chem. 24, 343-362. https://doi.org/10.1021/bc300419g.

[98] Yingyuad, P. et al. (2014) Enzyme-triggered PEGylated siRNA-nanoparticles for controlled release of siRNA. J. RNAi Gene Silenc. 10, 490-499. 
[99] Liu Y, et al. (2019) Tumor microenvironmental pH and enzyme dual responsive polymer-liposomes for synergistic treatment of cancer immuno-chemotherapy. Biomacromolecules 20, 882-892. https://doi.org/10.1021/acs.biomac.8b01510.

[100] Mizukami, S. et al. (2017) Enzyme-triggered compound release using functionalized antimicrobial peptide derivatives. Chem. Sci. 8, 3047-3053. https://doi.org/10.1039/c6sc04435b.

[101] Assanhou, AG. et al. (2015) Reversal of multidrug resistance by co-delivery of paclitaxel and lonidamine using a TPGS and hyaluronic acid dual-functionalized liposome for cancer treatment. Biomaterials 73, 284-295. https://doi.org/10.1016/j.biomaterials.2015.09.022.

[102] Lou, J., Best, M.D. (2020) A general approach to enzyme-responsive liposomes. Chem. Eur. J. 26, 8597-85607. https://doi.org/10.1002/chem.202000529.

[103] Lyu, D., Chen, S., Guo, W. (2018) Liposome crosslinked polyacrylamide/DNA hydrogel: A smart controlled-release system for small molecular payloads. Small 14, 1704039. https://doi.org/10.1002/smll.201704039.

[104] Zhu, L., Torchilin, V.P. (2013) Stimulus-responsive nanopreparations for tumor targeting. Integr. Biol. (Camb) 5, 96107. https://doi.org/10.1039/c2ib20135f.

[105] Cho, S.M., Lee, H.Y., Kim, J.C. (2008) pH-dependent release property of dioleoylphosphatidyl ethanolamine liposomes. Korean J. Chem. Eng. 25, 390-393. https://doi.org/10.1007/s11814-008-0066-6.

[106] Cullis, P.R., de Kruijff, B. (1978) The polymorphic phase behaviour of phosphatidylethanolamines of natural and synthetic origin. A 31P NMR study. Biochim. Biophys. Acta 513, 31-42. https://doi.org/10.1016/00052736(78)90109-8.

[107] Ellens, H., Bentz, J., Szoka, .FC. (1984) pH-Induced destabilization of phosphatidylethanolamine-containing liposomes: role of bilayer contact. Biochemistry 23,1532-1538. https://doi.org/10.1021/bi00302a029.

[108] Garg, A., Kokkoli, E. (2011) pH-Sensitive PEGylated liposomes functionalized with a fibronectin-mimetic peptide show enhanced intracellular delivery to colon cancer cell. Curr. Pharm. Biotechnol. 12, 1135-1143. https://doi.org/10.2174/138920111796117328.

[109] Slepushkin, V.A. et al. (1997) Sterically stabilized pH-sensitive liposomes. Intracellular delivery of aqueous contents and prolonged circulation in vivo. J. Biol. Chem. 272, 2382-2388. https://doi.org/10.1074/jbc.272.4.2382.

[110] Zhang, L. et al. (2015) High tumor penetration of paclitaxel loaded pH sensitive cleavable liposomes by depletion of tumor collagen $I$ in breast cancer. ACS Appl. Mater. Interfaces 7, 9691-9701. https://doi.org/10.1021/acsami.5b01473.

[111] Kanamala, M. et al. (2019) Dual pH-sensitive liposomes with low pH-triggered sheddable PEG for enhanced tumortargeted drug delivery. Nanomedicine (Lond) 14, 1971-1989. https://doi.org/10.2217/nnm-2018-0510.

[112] Chen, D. et al. (2011) Effects of a novel pH-sensitive liposome with cleavable esterase-catalyzed and pH-responsive double smart mPEG lipid derivative on ABC phenomenon. Int. J. Nanomedicine 6, 2053-2061. https://doi.org/10.2147/IJN.S24344.

[113] Chan, C.L. et al. (2012) Endosomal escape and transfection efficiency of PEGylated cationic liposome-DNA complexes prepared with an acid-labile PEG-lipid. Biomaterials 33, 4928-4935. https://doi.org/10.1016/j.biomaterials.2012.03.038.

[114] Xu, H. et al. (2015) Design and evaluation of pH-sensitive liposomes constructed by poly(2-ethyl-2-oxazoline)cholesterol hemisuccinate for doxorubicin delivery. Eur. J. Pharm. Biopharm. 91, 66-74. https://doi.org/10.1016/j.ejpb.2015.01.030.

[115] Yuba, E., Harada, A., Sakanishi, Y., Kono, K. (2011) Carboxylated hyperbranched poly(glycidol)s for preparation of $\begin{array}{llllll} & \mathrm{pH} \text {-sensitive liposomes. }\end{array}$ https://doi.org/https://doi.org/10.1016/j.jconrel.2010.03.001.

[116] Banerjee, S., Sen, K., Pal, T.K., Guha, S.K. (2012) Poly(styrene-co-maleic acid)-based pH-sensitive liposomes mediate cytosolic delivery of drugs for enhanced cancer chemotherapy. Int. J. Pharm. 436, 786-797. https://doi.org/10.1016/j.ijpharm.2012.07.059.

[117] Sugimoto, T. et al. (2017) Preparation of dual-stimuli-responsive liposomes using methacrylate-based copolymers with $\mathrm{pH}$ and temperature sensitivities for precisely controlled release. Colloids Surf. B Biointerfaces 155, 449-458. https://doi.org/10.1016/j.colsurfb.2017.04.043.

[118] Lee, J.M., Park, H., Oh, K.T., Lee, E.S. (2018) pH-responsive hyaluronated liposomes for docetaxel delivery. Int. J. Pharm. 547, 377-384. https://doi.org/10.1016/j.ijpharm.2018.06.028.

[119] Miyazaki, M. et al. (2019) Development of pH-responsive hyaluronic acid-based antigen carriers for induction of antigen-specific cellular immune responses. ACS Biomater. Sci. Eng. 5, 5790-5797. https://doi.org/10.1021/acsbiomaterials.9b01278. 
[120] Pekker, M., Shneider, P. (2015) Interaction between electrolyte ions and the surface of a cell lipid membrane. J. Phys. Chem. Biophys. 5. https://doi.org/10.4172/2161-0398.1000177.

[121] Chen, M.M. et al. (2018) pH-sensitive charge-conversional and NIR responsive bubble-generating liposomal system for synergetic thermo-chemotherapy. Colloids surf. B Biointerfaces 167, 104-114. https://doi.org/https://doi.org/10.1016/j.colsurfb.2018.04.001.

[122] Men, W. et al. (2020) Layer-by-layer pH-sensitive nanoparticles for drug delivery and controlled release with improved therapeutic efficacy in vivo. Drug Deliv. 27, 180-190. https://doi.org/10.1080/10717544.2019.1709922.

[123] Naziris, N. et al. (2020) pH-responsive chimeric liposomes: From nanotechnology to biological assessment. Int. J. Pharm. 574, 118849. https://doi.org/10.1016/j.ijpharm.2019.118849.

[124] Kitayama, H. et al. (2014) Thermotropic phase behavior of hydrogenated soybean phosphatidylcholine-cholesterol binary liposome membrane. Chem. Pharm. Bull (Tokyo) 62, 58-63. https://doi.org/10.1248/cpb.c13-00587.

[125] Obata, Y., Tajima, S., Takeoka, S. (2010) Evaluation of pH-responsive liposomes containing amino acid-based zwitterionic lipids for improving intracellular drug delivery in vitro and in vivo. J. Control Release 142, 267-276. https://doi.org/10.1016/j.jconrel.2009.10.023.

[126] Yaroslavov, A. et al. (2020) A novel approach to a controlled opening of liposomes. Colloids Surf. B Biointerfaces 190, 110906. https://doi.org/https://doi.org/10.1016/j.colsurfb.2020.110906.

[127] Liu, X. et al. (2012) Fliposomes: pH-triggered conformational flip of new trans-2-aminocyclohexanol-based amphiphiles causes instant cargo release in liposomes. J. Liposome Res. 22, 319-328. https://doi.org/10.3109/08982104.2012.698420.

[128] Veremeeva, PN. et al. (2014) pH-Sensitive liposomes with embedded 3,7-diazabicyclo[3.3.1]nonane derivative. Mendeleev Commun. 24, 152-153. https://doi.org/https://doi.org/10.1016/j.mencom.2014.04.008.

[129] Zheng, Y. et al. (2018) Fliposomes: trans-2-aminocyclohexanol-based amphiphiles as pH-sensitive conformational switches of liposome membrane - a structure-activity relationship study. Chem. Phys. Lipids 210, 129-141. https://doi.org/https://doi.org/10.1016/j.chemphyslip.2017.10.004.

[130] Deshayes, S., Morris, M.C., Divita, G., Heitz, F. (2005) Cell-penetrating peptides: tools for intracellular delivery of therapeutics. Cell Mol. Life Sci. 62, 1839-1849. https://doi.org/10.1007/s00018-005-5109-0.

[131] Jiang, T. et al. (2012) Dual-functional liposomes based on pH-responsive cell-penetrating peptide and hyaluronic acid for tumor-targeted anticancer drug delivery. Biomaterials 33, 9246-9258. https://doi.org/10.1016/j.biomaterials.2012.09.027.

[132] Zhao, Y. et al. (2016) Tumor-specific pH-responsive peptide-modified pH-sensitive liposomes containing doxorubicin for enhancing glioma targeting and anti-tumor activity. J. Control Release 222, 56-66. https://doi.org/https://doi.org/10.1016/j.jconrel.2015.12.006.

[133] Shi, K. et al. (2015) A pH-responsive cell-penetrating peptide-modified liposomes with active recognizing of integrin av 33 for the treatment of melanoma. J. Control Release 217, 138-50. https://doi.org/10.1016/j.jconrel.2015.09.009.

[134] Han, Q. et al. (2016) Switchable liposomes: targeting-peptide-functionalized and pH-triggered cytoplasmic delivery. ACS Appl. Mater. Interfaces 8, 18658-18663. https://doi.org/10.1021/acsami.6b05678.

[135] Oumzil, K. et al. (2015) pH-cleavable nucleoside lipids: A new paradigm for controlling the stability of lipid-based delivery systems. ChemMedChem 10, 1797-17801. https://doi.org/10.1002/cmdc.201500381.

[136] Zhan, L. et al. (2017) A new type of pH-sensitive phospholipid. Tetrahedron Lett. 58, 4724-4727. https://doi.org/https://doi.org/10.1016/j.tetlet.2017.11.013.

[137] Liskayová, G. et al. (2019) pH-sensitive $N, N$-dimethylalkane-1-amine $N$-oxides in DNA delivery: From structure to transfection efficiency. Langmuir 35, 13382-13395. https://doi.org/10.1021/acs.langmuir.9b02353.

[138] Brown, J.M., Giaccia, A.J. (1998) The unique physiology of solid tumors: Opportunities (and problems) for cancer therapy. Cancer Res. 58, 1408-1416.

[139] Horsman, Mz.R. et al. (2012) Imaging hypoxia to improve radiotherapy outcome. Nat. Rev. Clin. Oncol. 9, 674-687. https://doi.org/10.1038/nrclinonc.2012.171.

[140] Wouters, A., Pauwels, B., Lardon, F., Vermorken, J.B. (2007) Review: Implications of in vitro Rresearch on the Effect of radiotherapy and chemotherapy under hypoxic conditions. Oncologist 12, 690-712. https://doi.org/10.1634/theoncologist.12-6-690.

[141] Swartz, M.A. et al. (2012) Tumor microenvironment complexity: Emerging roles in cancer therapy. Cancer Res. 72, 2473-2480. https://doi.org/10.1158/0008-5472.CAN-12-0122.

[142] Feng, L. et al. (2017) Theranostic liposomes with hypoxia-activated prodrug to effectively destruct hypoxic tumors post-photodynamic therapy. ACS Nano 11, 927-937. https://doi.org/10.1021/acsnano.6b07525. 
[143] Liu, H. et al. (2017) Development of a hypoxia-triggered and hypoxic radiosensitized liposome as a doxorubicin carrier to promote synergetic chemo-/radio-therapy for glioma. Biomaterials 121, 130-143. https://doi.org/10.1016/j.biomaterials.2017.01.001.

[144] Li, Y. et al. (2019) Nitroimidazole derivative incorporated liposomes for hypoxia-triggered drug delivery and enhanced therapeutic efficacy in patient-derived tumor xenografts. Acta Biomater. 83, 334-348. https://doi.org/10.1016/j.actbio.2018.10.029.

[145] Long, M. et al. (2020) Azo-inserted responsive hybrid liposomes for hypoxia-specific drug delivery. Acta Biomater. 115, 343-357. https://doi.org/10.1016/j.actbio.2020.07.061.

FIGURE 1. Schematic representation of the main chemical strategies employed for the development of redox-sensitive LPs.

FIGURE 2. Redox-responsive chemical structures 1-4, involved in GSH-mediated LP deshielding.

FIGURE 3. a) Cationic responsive lipids employed in combination with phospholipids to afford multilamellar LP-DNAcomplex self-assembled structures; b) tocopherol-based redox-sensitive cationic lipids as building blocks in LP formation; c) redoxresponsive pyropheophorbide $\alpha$-lipid conjugate; d) Prodrugs sensitive to redox environments; e) mechanistic insights into redox-triggered drug release.

FIGURE 4. GSH-activated self-reporting constructs and lipoplexes: structures and activation mechanism.

FIGURE 5. a) Payload release triggered by reduction of quinone $\mathbf{1 8}$ and intramolecular cyclization of hydroquinone to yield an inverted-phase-forming DOPE and a lactone; b) ferrocene-based phospholipids 19a, b and lipid 19c, exploiting Fe" to Fe ${ }^{\text {III }}$ oxidation; c) polymerization between a thiol aryl PEG and maleimide groups present on the surface of assembled LPs.

FIGURE 6. a) PLA2-sensisitve phospholipids used as building blocks in common LP formulations; b) schematic representation of PLA2 triggered drug-release.

FIGURE 7. PLA2-mediated hydrolysis and activation of PLA2-targeted prodrugs where payloads are covalently bound to modified phospholipids at the sn-2 position.

FIGURE 8. Sequential PLA2- and PLD-mediated hydrolysis and activation of payloads covalently bound to modified phospholipids.

FIGURE 9. a) Protease-sensitive constructs (cleavable sequences in red) assembled with other phospholipids to afford protease-sensitive LPs; b) bare LPs decorated with a MMP-2 sensitive polymer.

FIGURE 10. Key MMP-sensitive molecules used in targeted LPs deshielding via: a) pre-assembly functionalization; b) postassembly through the maleimide-thiol reaction; c) MMP2- and pH-responsive LPs for efficient delivery of payload drugs.

FIGURE 11. a) TPGS- and HA-centered, dual-functionalized LPs suitable for HAase-triggered HA degradation; b) structure and mechanism of action of enzyme-sensitive lipids targeted against enzymes other than phospholipases and proteases.

FIGURE 12. Chemical structures of $\mathrm{pH}$-sensitive liposomes components 43-56 (a light blue color indicates the $\mathrm{pH}$ sensitive portion of each construct).

FIGURE 13. Conformational switch of flipid tails at mild acidic $\mathrm{pH}$ (a blue color indicates their pH sensitive portion).

FIGURE 14. Chemical structures of unnatural lipids 59-62 used for the preparation of pH-sensitive liposomes (a light blue color indicates their $\mathrm{pH}$-sensitive portions).

FIGURE 15. Chemical structures of bioreductive prodrug 63a, of nitroimidazole-based 64a, 65a of Si-based construct 66a used for the preparation of hypoxia-responsive LPs, and of their corresponding reduced derivatives (63-66)b (a blue color indicates their hypoxia-sensitive portions). 


\section{Biosketches}

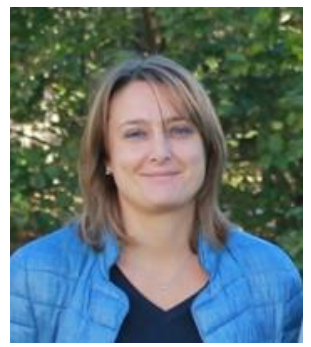

Sara Pellegrino is Associate Professor of Organic Chemistry at the University of Milano, Department of Pharmaceutical Sciences, Italy. Her research focus on the synthesis of peptides and peptide mimics, and their application in Medicinal Chemistry, Biochemistry and Material Science. Prof Pellegrino is particularly interested in peptide self-assembly and in deciphering molecular interactions at the basis of peptides activities, both as bioactive compounds and as biocomponents for drug delivery. Her studies take advantage on the use of non-standard amino acids as molecular bricks for the stabilization of peptide conformation and activity.

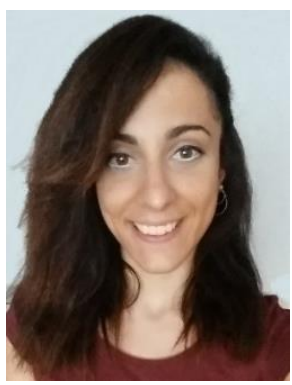

Sabrina Giofrè is a post-doc researcher in the group of Prof. Pierfausto Seneci. She completed her PhD in Pharmaceutical Science at Università degli Studi di Milano in 2020, under the supervision of Prof. Egle Beccalli. Her research is mostly focused on the synthesis of compounds with biological significance, exploiting transition metal-catalysis. Lately, her research interests also involve the chemical synthesis of modified peptides and phospholipids as self-assembly molecules for drug delivery.

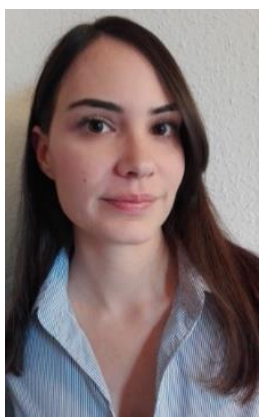

Antonia Antoniou is a postdoctoral research associate at the University of Milan, in the group of Professor Pierfausto Seneci. Her fields of interest include organic synthesis, solid phase synthesis, chemical modifications, and spectroscopic characterization. After MSc in Medicinal Chemistry, she completed her PhD in 2017 in Organic Chemistry and currently she is working in the NEVERMIND project for the synthesis of oligopeptides and phospholipids, as well as their synthetic analogues, peptidomimetics and modified phospholipids. 


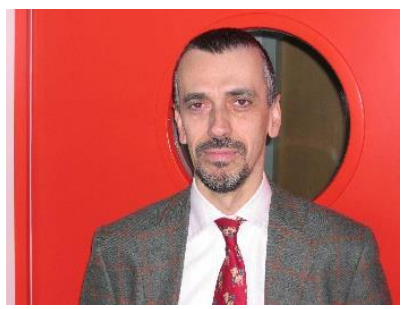

Pierfausto Seneci is Associate Professor of Organic Chemistry at the University of Milano, Department of Chemistry, Italy. His research focus on Medicinal Chemistry applied to the rational design and synthesis of anticancer and neuroprotective compounds. Prof Seneci is particularly interested in the study of misfolding diseases through chemical probes for photoactivation and affinity chromatography studies, and in the rational design of small molecules acting against immuneoncological targets.

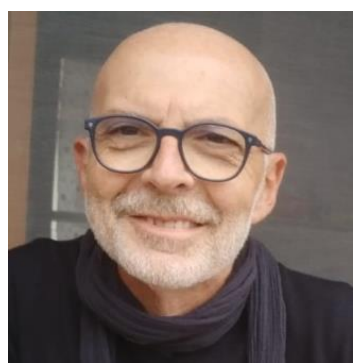

Daniele Passarella is Full Professor of Organic Chemistry at the University of Milano, Department of Chemistry, Italy. His research interest is focused on Organic and Bioorganic Chemistry with particular attention toward the preparation of new anti-cancer and anti-neurodegenerative compounds. One of the main topics regards the preparation of conjugate compounds for the obtainment of self-assembled nanoparticles. 